CU-PHYSICS/06-2008

HIP-2008-11/TH

TIFR-TH-08-12

\title{
Universal Extra Dimensions, Radiative Returns and the Inverse Problem at a Linear $e^{+} e^{-}$Collider
}

\author{
Biplob Bhattacherjee ${ }^{a}$, Anirban Kundu ${ }^{a}$, Santosh Kumar Rai ${ }^{b}$, and Sreerup Raychaudhuri $c 1_{1}$ \\ ${ }^{a}$ Department of Physics, University of Calcutta, \\ 92, Acharya Prafulla Chandra Road, Kolkata 700 009, India. \\ E-mail: bbhattacherjee@gmail.com, akphy@caluniv.ac.in \\ ${ }^{b}$ Department of Physics, University of Helsinki and Helsinki Institute of Physics, \\ P.O. Box 64, FIN-00 014, University of Helsinki, Finland. \\ E-mail: santosh.rai@helsinki.fi \\ ${ }^{c}$ Department of Theoretical Physics, Tata Institute of Fundamental Research, \\ 1, Homi Bhabha Road, Mumbai 400 005, India. \\ E-mail: sreerup@theory.tifr.res.in
}

\begin{abstract}
In a five-dimensional model with one Universal Extra Dimension (UED), signals for a pair of $n=1$ Kaluza-Klein excitations could be easily observed at a future $e^{+} e^{-}$collider if the process in question is kinematically allowed. However, these signals would prove difficult to distinguish from those predicted in other models, such as those with an extended gauge symmetry or supersymmetry. A much better power of discrimination is provided by the fact that the same machine could also produce the $n=2$ gauge bosons $\gamma_{2}$ and $Z_{2}$ as resonances in fermion pair-production without any upgrade in the collision energy $\sqrt{s}$. Assuming a fixed $\sqrt{s}$ - as is expected at upcoming $e^{+} e^{-}$machines - we investigate the role of beam radiation in helping to excite such resonances through radiative returns. We then show how these resonances could yield unambiguous signals for UED, if taken in conjunction with the production of $Z_{1}$ pairs, identified by their decays to leptons and missing transverse energy.
\end{abstract}

PACS numbers: $11.10 . \mathrm{Kk}, 12.60 . \mathrm{Cn}, 13.66 . \mathrm{Hk}$

October 31, 2018

\footnotetext{
${ }^{1}$ On leave of absence from the Department of Physics, Indian Institute of Technology, Kanpur 208016, India.
} 


\section{Introduction}

The last decade has seen a remarkable revival of interest in phenomenological theories having extra spacetime dimensions - an idea which goes all the way back [1] to Nørdstrom (1914), Kaluza (1919) and Klein (1926). Today we have a number of such models, which differ on many specific counts, such as the number of extra dimensions, the geometry of spacetime, and the choice of which fields to keep confined within the four canonical Minkowski dimensions while others are permitted to go into the extra dimensions or 'bulk'. A generic feature of all these extra dimensional models is the fact that many of these detailed features are decided ad hoc rather than derived from some underlying principle - the hope being that a more fundamental theory, when revealed, would provide the necessary dynamics. In this work, we focus on the Universal Extra Dimension (UED) model proposed by Appelquist, Cheng, and Dobrescu [2], which has one extra compact dimension and all the fields of the Standard Model (SM) are defined over the bulk. In such models, the compact extra dimension(s) cannot form a simple manifold like the circle, sphere or torus considered in traditional Kaluza-Klein theories [3], because that would not be able to support the chiral fermions known to be present in the SM and hence would not permit parity-violation. A more exotic topology is, therefore, required. In the simplest UED scenario - to which we confine ourselves - there is only one extra dimension, denoted by $y$, but this is compactified on a $\mathbb{S}_{1} / \mathbb{Z}_{2}$ orbifold, i.e. a circle of radius $R$ folded about one of its diameters. Mathematically, this means that we simultaneously impose two symmetries, viz. $y \rightarrow y+2 \pi R$ and $y \rightarrow-y$. This 'orbifolding' is sufficient to provide the necessary distinction between chiral components of fermions.

The particle phenomenology of UED models is based on the following major features [2, 4]:

- When projected in four spacetime dimensions, every bulk field $\Psi_{P}\left(x^{\mu}, y\right)$ is associated with a tower of Kaluza-Klein (KK) excitations $P_{0}, P_{1}, P_{2}, \ldots$ The mass of $P_{n}$ is given, at the tree-level, by

$$
M_{n}^{2}=M_{0}^{2}+\frac{n^{2}}{R^{2}}
$$

where $n=0,1,2, \ldots$ and $M_{0}$ is the mass of the SM particle $P_{0}$.

- Since all the fields can access the bulk, momentum is expected to be conserved along the fifth compact direction, and hence the KK number $n$ is conserved. To get chiral fermions, we need the $\mathbb{Z}_{2}$ symmetry $y \rightarrow-y$ which breaks the translational symmetry along $y$ and induces terms located at the orbifold fixed points $y=0$ and $y=\pi R$. Obviously, such terms will violate KK-number conservation, though we can expect such effects to be suppressed by the boundary-to-bulk ratio. On the other hand, since there is no physical difference between the two fixed points at $y=0$ and $y=\pi R$, 
we obtain a residual $\mathbb{Z}_{2}$ symmetry $y \rightarrow y+\pi R$. The outcome, so far as the effective interaction Lagrangian after compactification goes, is the conservation of KK-parity defined as $(-1)^{n}-$ at every interaction vertex.

- The terms located at the orbifold fixed points are logarithmically divergent, i.e. they are proportional to $\log \Lambda^{2}$ where $\Lambda \gg R^{-1}$ is the scale up to which the theory is expected to be valid.

- Thus, the minimal UED model has two free parameters: $R$ and $\Lambda$, in addition to the mass $M_{H}$ of the SM Higgs boson. It is convenient to carry out phenomenological studies of the UED model with reference to the plane defined by $R^{-1}$ (dimension of mass) and $\Lambda R$ (dimensionless) as Cartesian axes. This constitutes the 'theory space' of the UED model.

- While the tree-level masses of the KK excitations follow the formula given in Eq. (1), important changes are observed at the one-loop level, where quantum numbers like spin, flavour and colour begin to play a role. In fact, these corrections control the mass-splittings and mixing angles between states (and hence allowed transitions) for any given excitation level with $n \neq 0$. Since many of the radiative corrections at the one-loop level are logarithmically divergent, this introduces a critical dependence of the phenomenological effects on $\Lambda$ at every level except $n=0$.

- Since KK-parity is conserved, the lightest $n=1$ particle must be stable. In the minimal UED model, this is almost always the $\gamma_{1}$ - the $n=1$ analogue of the photon. It turns out that this is more-or-less identical with the $n=1$ excitation of the hypercharge gauge boson $B$, since the 'Weinberg angles' for all $n \neq 0$ levels turn out to be small. The $\gamma_{1}$ is an excellent cold dark matter candidate - in fact, this is one of the most attractive features of the UED model. In a collider experiment, this Lightest KK Particle (LKP) is 'invisible' because of its weak interaction with matten2, leading to a characteristic signature with large amounts of missing energy and momentum.

- All $n=1$ particles will undergo decays which eventually cascade down to the LKP. However, as the mass splitting among the $n=1$ states is generally small (being induced by radiative corrections), these cascade decays will generally yield one (or more) soft lepton(s) or jet(s) associated with a large missing $p_{T}$ due to the LKP.

\footnotetext{
${ }^{2}$ Because of conservation of KK parity, the interaction of a $\gamma_{1}$ with ordinary matter can only be by exchange of an $n=1$ (or higher) particle, and hence the cross-section is suppressed by the large masses of these excitations. This is somewhat similar to the way in which neutrino-matter interactions are suppressed by the large mass of the exchanged $Z$ boson.
} 
The close resemblance of this phenomenology with that of supersymmetry (SUSY) - in its usual $R$-parity conserving avatar - is immediately obvious. All $n=0$ excitations are identical with the $R_{p}=1$ particles of the SM, while all $n=1$ excitations are analogous to the $R_{p}=-1$ super-partners. Conservation of KK-parity plays the same role as conservation of R-parity: the stable LKP behaves exactly like the stable LSP (lightest SUSY particle) in leading to missing energy and momentum signals. UED signals could, therefore, easily be mistaken for SUSY signals and vice versa [5]. It is true that in the case of UEDs, the spin of the $n>0$ excitations is the same as that of their $n=0$ counterparts, but this is more a matter of detail, where the phenomenological effects are concerned. It is, therefore, a valid - perhaps crucial - question to ask if a new physics signal with the expected features is due to a underlying UED or to a SUSY theory, and to look for ways to confirm this.

One important difference between SUSY and UED models immediately springs to the eye. This is the fact that in UED models, instead of one set of heavy partners of the SM particles, there is a whole tower of such partners for each SM particle. Thus, if we could pair-produce not just the $n=1 \mathrm{KK}$ modes, but also the $n=2 \mathrm{KK}$ modes, and find their masses to be in the expected ratio (approximately 1:2, modulo radiative corrections), that would constitute very strong circumstantial evidence [5] for UED. Pair-production of $n=2$ resonances would be a simple matter, if we had at our disposal a machine with arbitrarily large energy, but in practice, we only have machines with a limited kinematic access. In fact, the current experimental lower bounds on $R^{-1}$ of around $300-400 \mathrm{GeV}$ already make it problematic to pair-produce the $n=2$ states at existing and planned colliders, including the Large Hadron Collider (LHC). One can still try to differentiate $n=1$ UED signals from those of SUSY models by trying to identify the spin of the intermediate states from the angular distribution of the decay products [6]. This could, however, be a tricky business, requiring the reconstruction of the rest frame of the decaying particles in the presence of large amounts of missing momentum.

There is, however, one saving grace, and this is the fact that the $n=2$ modes can couple singly to a pair of the $n=0$ modes (i.e. SM particles), since all of them have positive KK-parity. It is thus possible, for example, to have $\gamma_{2}$ and $Z_{2}$ resonances in a four-fermion process, where both the initial and final di-fermion states are purely $(n=0)$ SM particles. Since the masses of these KK modes satisfy $M_{\gamma_{2}} \approx 2 R^{-1} \approx 2 M_{\gamma_{1}}$ and $M_{Z_{2}} \approx 2 R^{-1} \approx 2 M_{Z_{1}}$, the resonance energy is more or less the same as that required to pair-produce $\gamma_{1}$ 's or $Z_{1}$ 's. Thus, if one can reach this energy threshold, one could, in principle, obtain much stronger evidence for UED by demanding the simultaneous presence of these resonances with the signals from pair production of the $n=1 \mathrm{KK}$ states. The obvious place to look for such $s$-channel resonances would be in the LHC data [7], which are expected to start coming in soon. Unfortunately, however, the $\gamma_{2}$ and $Z_{2}$ resonances can be shown to decay almost 
exclusively into jets 3 (without any missing energy). Any signal with a pair of jets is sure to be completely lost against the enormous QCD background at the LHC. One must, then, turn to a high-energy $e^{+} e^{-}$collider, such as the proposed ILC, to observe these resonances in dijet production. Here, the production cross-section would be suppressed by the smallness of the $\gamma_{2} e^{+} e^{-}$or $Z_{2} e^{+} e^{-}$coupling, but this disadvantage can be largely offset by enhancement due to resonant effects and the high luminosity expected at such a machine. Obviously, dijet final states have a much smaller background at an $e^{+} e^{-}$machine, making them viable for new physics searches.

(a)

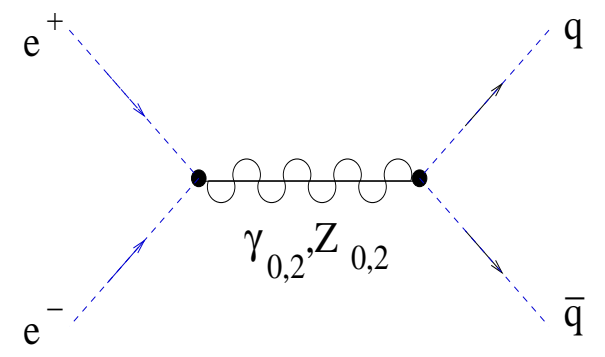

(b)

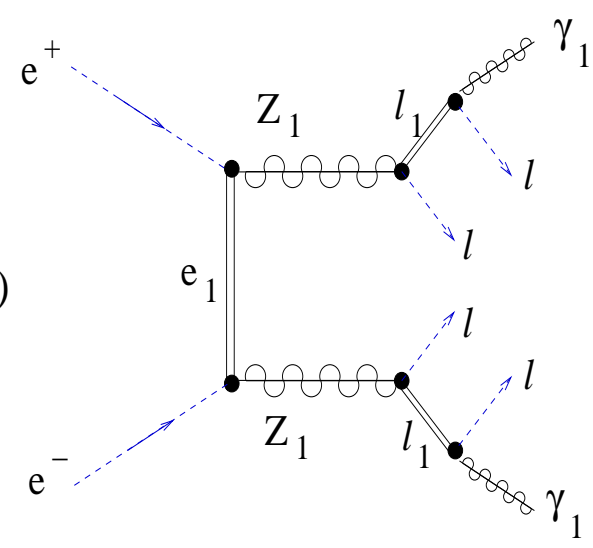

Figure 1: Feynman diagrams showing the most important processes involving KK excitations at an $e^{+} e^{-}$ collider. The upper graph, marked (a), illustrates s-channel exchange of $\gamma, Z$ and $\gamma_{2}, Z_{2}$, which add coherently. The lower graph, marked (b), illustrates pair production of $Z_{1}$ excitations, which also has a crossed diagram (not shown in the figure). Leptonic decays of the $Z_{1}$ are also illustrated, with the symbol $l$ generically standing for any charged lepton or neutrino. Dashed (blue) lines indicate SM particles.

In Figure 1, we show some of the major Feynman diagrams contributing to UED signals at a high-energy $e^{+} e^{-}$collider. The upper diagram, marked (a), shows dijet production

\footnotetext{
${ }^{3}$ We note that the leptonic branching ratios of the $n=2$ excitations of $\gamma$ and $Z$ are much smaller than the leptonic branching ratios of an ordinary $(n=0) Z$ boson. It may still be interesting to carry out a search for the resonant contributions to Drell-Yan dileptons at the LHC, though the smallness of the excess cross-section could make such delicate searches difficult (and inaccurate) in the messy environment of a hadron collider $[8]$.
} 
with exchange of real or virtual KK excitations (bearing even KK number) of the photon or $Z$-boson, while the lower one, marked (b), shows pair-production of $Z_{1}$ excitations, leading to a final state with $4 \ell+\mathbb{E}_{T}$. An earlier study [9] has shown that resonant production, as indicated in Figure 1 $(a)$ is indeed a viable possibility, with strong resonances being obtained when the machine energy $\sqrt{s}$ coincides with the poles of the $\gamma_{2}$ and/or $Z_{2}$ propagators. However, the analysis in Ref. [9] makes the assumption that signals for UED would be discovered at the LHC, leading to an approximate knowledge of the masses of $\gamma_{1}$ and $Z_{1}$ and hence, of the compactification parameter $R^{-1}$. Knowing this parameter - and therefore, the masses $M_{\gamma_{2}}$ and $M_{Z_{2}}$-it was assumed that the centre-of-mass energy $\sqrt{s}$ of the $e^{+} e^{-}$ collider would be tuned to these resonances, in the same way as LEP-1 had been tuned to the $Z_{0}$ resonance after its discovery at the UA1/UA2.

In this context, one of the most often-quoted pieces of folklore in high energy physics is the statement that the next high-energy $e^{+} e^{-}$collider - presumably the planned International Linear Collider (ILC) - will bear the same relation to the LHC as the LEP experiment bore to the UA1/UA2, i.e. if a resonant state is found at the LHC, precision measurements of that state would be made at the ILC. This is, in essence, the assumption made in Ref. [9]. However, there is a crucial difference between the planning of LEP and the ILC, viz. the fact that LEP-1 was tuned to the $Z$ resonance, which had already been discovered at the UA2, whereas the ILC is being planned even before the LHC has commenced its run. According to this plan, the ILC will operate at fixed energies of $\sqrt{s}=500 \mathrm{GeV}$, with a later upgrade to $\sqrt{s}=1 \mathrm{TeV}$. It would, therefore, be entirely a matter of luck if the pole of a resonant state happens to lie at (or close to) these two values of the collision energy. A much more likely scenario, however, would have the resonance in question lying several decay widths away from the machine energy, in which case it will not show up as a signal of any significance. This is as true of the Kaluza-Klein resonances of the UED model as of other exotic particles like an extra $Z^{\prime}$ or a massive graviton, and indeed of the SM $Z$ boson itself.

All is not lost, however, for a way to observe these states will be provided by Nature herself. The discovery of resonant states far away from the machine energy $\sqrt{s}$ still remains possible, because of the well-known phenomenon of 'radiative returns' at an $e^{+} e^{-}$machine, which was first observed in the LEP-1.5 runs at $\sqrt{s}=130-136 \mathrm{GeV}$. There, the occasional emission of a hard collinear photon with energy $E_{\gamma}$ from the initial electron/positron state had led to a corresponding degradation of the effective centre-of-mass energy to $\sqrt{\hat{s}}=\sqrt{s}-E_{\gamma}$. During the LEP-1.5 run, there were a small number of such events where the energy was sufficiently reduced to match the $Z^{0}$-pole around $\sqrt{\hat{s}}=M_{Z} \simeq 91 \mathrm{GeV}$, and the large resonant crosssection around the pole ensured that this extra contribution to the total $e^{+} e^{-}$cross-section became quite substantial. 
In the LEP-1.5 runs, the radiation from the beam constituents, i.e. electron/positron occurred because of the strong electromagnetic fields inside a bunch of electrons as it moved through the beam pipe. This effect, called initial state radiation, or ISR, is always present and can be predicted fairly accurately using QED alone. At the ILC, this ISR effect will certainly be present, but it will also be accompanied by another effect, viz. beamstrahlung, which is the emission of photons from the initial state electron/positron just before a collision under the influence of the electromagnetic fields of the other colliding bunch. This second QED effect was very weak at the LEP because the number density of charged particles in a bunch was rather low compared to the projected number density at the ILC. A low bunch density was made possible at the LEP because the design luminosity was easily attainable by multiple collisions (some 2000 per bunch) in the storage ring geometry. At a single-pass linear collider, however, the higher luminosity requirement demands tightly-packed bunches generating strong electromagnetic fields. To cut a long story short, therefore, radiative returns at the ILC will be a combined effect due to both ISR and beamstrahlung, and will create a substantial spread in the energy of the colliding beams. Turning this fact to our advantage, we can claim that even in the case of a fixed-energy ILC (or any other highenergy $e^{+} e^{-}$machine), such radiative returns could well be the discovery mechanism of such resonances [10]. The rarity of a radiative return (the probability is suppressed by the QED coupling $\alpha$ ) would be adequately offset by the large resonant cross-section at the pole of the propagator.

Coming back to the UED model, we have already noted that the energy $E_{Z_{1} Z_{1}}$ required to pair-produce the $Z_{1}$ is around $E_{Z_{1} Z_{1}} \approx 2 R^{-1}$, which is the same as the energy required for resonance production of $\gamma_{2}$ or $Z_{2}$, both of which have masses close to $2 R^{-1}$. Thus, if $R^{-1}$ is small enough for these states to be accessible to an $e^{+} e^{-}$machine (radiative returns and all), it would be quite feasible to carry out a simultaneous study of the $4 \ell+\mathbb{E}_{T}$ signal from a $Z_{1} Z_{1}$ pair as well as the dijet signals from resonant $\gamma_{2}$ and $Z_{2}$ states. If we find a significant effect in both these channels, it can be argued quite convincingly that the circumstantial evidence is strong enough to pin down a unique signal for UED - one which would discriminate it not only from the SM but also from SUSY, massive gravitons, extra gauge bosons, etc. in other rival models going beyond the SM.

At this point, one may pause to reflect on the $Z_{1}$ pair production process and note that the dominant decay $Z_{1} \rightarrow \ell \bar{\ell} \gamma_{1}$ includes both charged leptons and neutrinos under the generic symbol $\ell$. It is possible, therefore, for a $Z_{1}$ to decay into a $\nu \bar{\nu} \gamma_{1}$ final state, which would be completely invisible. There are, therefore, three distinct possibilities:

\footnotetext{
${ }^{4}$ This unavoidable energy spread is among the reasons why the ILC design envisages a pre-determined machine energy rather than one tuned to a possible resonance.
} 
1. Both $Z_{1}$-s decay to charged lepton pairs and missing energy - the signal has four charged lepton tracks with unbalanced (transverse) momentum;

2. One $Z_{1}$ decays to a pair of charged leptons and missing energy, the other decays invisibly - the signal has two charged lepton tracks with unbalanced (transverse) momentum; and

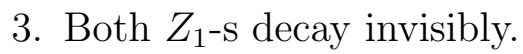

The last possibility may be immediately discounted, since there would be nothing to trigger on. We shall see, presently, that the leptons coming from the $Z_{1}$ decay are relatively soft, with $p_{T}<100 \mathrm{GeV}$ in general. This means that for the second option, viz. a dilepton with missing energy, there will be a significant background from soft processes tending to produce dileptons, such as two-photon processes, vector boson fusion, etc. There will even be some background from decays of soft hadrons which would normally be dismissed as noise in the hadron calorimeter. It is best, therefore, to focus on the first option, viz. $e^{+} e^{-} \rightarrow \ell^{+} \ell^{+} \ell^{-} \ell^{-}+\mathbb{E}_{T}$, which would have relatively little soft background, and can be clearly distinguished from other hard processes by the low $p_{T}$ of the detected leptons.

The thrust of this work is, therefore, twofold. The first part is to show that radiative returns caused by ISR and beamstrahlung effects can excite the $\gamma_{2}$ and $Z_{2}$ resonances in $e^{+} e^{-}$collisions and provide observable effects, even when their masses are far away from the machine energy. The second part is to show how one can combine these with the signals for $Z_{1}$ pair-production at the same machine to identify a potential new physics discovery as specifically due to UED. As both dijets and $4 \ell+\mathbb{E}_{T}$ will certainly be among the final states which would be studied anyway when the ILC or any other high energy $e^{+} e^{-}$collider becomes operational, the present study suggests an economical and accurate test for UED at such a machine.

This article is organised in the following manner. In Section 2, we describe some details of the UED model which are relevant to the phenomenological studies which follow. This is followed in Section 3 by a brief introduction to the technique used to estimate the level of ISR and beamstrahlung. Our results on resonance production with radiative returns are presented and analysed in Section 4, while Section 5 describes how this can be used in conjunction with $Z_{1}$ pair production to identify UED signals uniquely. Section 6 contains a brief summary and a few further comments of a general nature. 


\section{The UED Model}

It has already been mentioned that in UED models, all the SM fields live in a $\mathbb{R}_{4} \times\left(\mathbb{S}_{1} / \mathbb{Z}_{2}\right)$ spacetime, of which $\mathbb{R}_{4}$ is the canonical Minkowski space, and the remaining spatial dimension corresponds to a circle folded about a diameter. Naturally, all the particle momenta in the UED model will have five components, of which the component along the compact fifth spatial dimension has to be discrete, increasing in steps of size $R^{-1}$. Compactification to four dimensions then yields a Kaluza-Klein (KK) tower of states for every field, with the discrete fifth component of momentum appearing as an extra contribution to the mass $M_{0}$ of the zero mode, as shown in Eq. (11). Apart from the zero mode (identified with the SM particle), the other KK excitations are expected to be heavy if the parameter $R^{-1}$ is chosen large enough.

The value of $R^{-1}$, i.e. the size of the extra compact dimension, is of paramount importance in UED phenomenology. Obviously, it cannot be too low, else there would already be experimental evidence for KK excitations. As a matter of fact, current experimental data already seem to prefer $R^{-1}$ to be in the range of a few hundreds of $\mathrm{GeV}$. On the other hand, if $R^{-1}$ is too high, the $\mathrm{KK}$ excitations would be beyond the kinematic reach of upcoming collider experiments, a scenario which, though not impossible, would be very disappointing. Fortunately, however, there is a cosmological argument which militates strongly in favour of a value of $R^{-1}$ which would keep the lowest KK excitations within the range accessible to terrestrial experiments. We have already explained in the last section that KK-parity $(-1)^{n}$ has to be conserved and that this makes the LKP stable and weakly interacting, i.e. an excellent candidate for the cold dark matter (CDM) component of the Universe. Assuming, then, that the CDM is entirely composed of LKP's, we can take $\Omega_{\mathrm{CDM}}=0.110 \pm 0.006 h^{-2}$ for the density of cold dark matter and obtain [11] an upper bound5 on $R^{-1}$ of about 900 $\mathrm{GeV}$. This is well within the kinematic reach of planned and upcoming colliders - of which the LHC is the prime example. However, it is only fair to say that this bound depends on two assumptions, viz. that there exists a substantial relic density of LKP-s and that there is no other component of cold dark matter. These assumptions are reasonable, but not, of course, absolutely essential. In this article, we accept them as binding and hence we focus on the ILC, which can explore up to about $R^{-1}=450 \mathrm{GeV}$ and the CLIC, the proposed multi-TeV $e^{+} e^{-}$machine at CERN, Geneva, which can explore the entire range up to $R^{-1}=900 \mathrm{GeV}$.

Complementing the cosmological upper bound, a number of low- and intermediate-energy processes constrain the parameter $R^{-1}$ at the lower end [12, 13, 14]. For example, the non-

\footnotetext{
${ }^{5}$ This can go down as far as $600 \mathrm{GeV}$ in the eventuality that all three generations of $\mathrm{SU}(2)$ singlet $n=1$ leptons are nearly mass-degenerate (within 1\%) with the LKP $\gamma_{1}$. However, as this is only true for extreme choices of $\Lambda R$, we prefer the more conservative limit of $900 \mathrm{GeV}$.
} 
observation of KK modes at colliding-beam machines like the LEP, Tevatron and HERA immediately tells us that the $n \neq 0$ excitations are beyond the kinematic reach of these machines, i.e. the lower bound on $R^{-1}$ is pushed up to at least $150 \mathrm{GeV}$. However, a much more stringent bound comes [13] from the radiative decay $B \rightarrow X_{s} \gamma$. Taking both experimental error and theoretical uncertainties at the $5 \sigma$ level and making allowances for uncertain elements in QCD corrections to the new physics contribution, the current data on $B \rightarrow X_{s} \gamma$ roughly translates to a firm lower bound $R^{-1} \gtrsim 300 \mathrm{GeV}$. Electroweak precision observables are also quite sensitive to $R^{-1}$, but the actual bound obtained is dependent on the mass $M_{H}$ of the Higgs boson. If $M_{H}$ is low $(\sim 115 \mathrm{GeV})$, then the precision data severely restrict $R^{-1}$ to $R^{-1} \gtrsim 600 \mathrm{GeV}$ at $99 \%$ confidence level, but this bound will fall to about $400 \mathrm{GeV}$ if the Higgs boson turns out to be heavy $\left(M_{H} \sim 350 \mathrm{GeV}\right)$ and even as low as $300 \mathrm{GeV}$ if $M_{H}$ is as large as $500 \mathrm{GeV}$ [14]. Taking the widest range into account, therefore, our study will consider $R^{-1}$ varying in the range $300 \mathrm{GeV} \lesssim R^{-1} \lesssim 900 \mathrm{GeV}$.

The formula for the mass of a KK excitation exhibited in Eq. (11) gets modified once we take into account the radiative corrections in a quantum field theory. Of course, like all such theories in higher dimensions, the UED model is non-renormalisable and must be treated in the spirit of an effective theory, valid up to a cutoff scale $\Lambda \gg R^{-1}$ - at which point one must begin to consider an explicitly five-dimensional theory. Radiative corrections to the masses of the KK particles have been computed in Refs. [4], [15] and [16], where it has been shown that for any KK level $(n>0)$, the almost-mass-degenerate spectrum resulting from Eq. (11) splits up due to such correction terms. There are, in fact, two classes of corrections, viz.

- The bulk corrections: These correspond to loop diagrams that are sensitive to compactification. The bulk corrections are small - identically zero for fermions - and hardly play an important role in determining the mass spectrum.

- The boundary corrections: These terms are related with the interactions that are present only at the orbifold fixed points. They are much larger than the bulk corrections and are divergent, growing as $\log \Lambda$. It is these boundary corrections which play a major role in determining the exact spectrum (and hence possible decay modes) of the KK excitations for $n>0$.

Once the mass spectrum and the interactions are determined, one can proceed to make a phenomenological study of the UED model. Studies of the low-energy phenomenology of this model may be found in Refs. 22], [12], [13] and [14], while high-energy collider signatures are discussed in Refs. 99, [17], [18] and [19]. Much of this work may be summed-up and absorbed in the choice of range $300 \mathrm{GeV} \lesssim R^{-1} \lesssim 900 \mathrm{GeV}$ for the size of the compact dimension. To get concrete predictions we choose two benchmark values $R^{-1}=400 \mathrm{GeV}$ 
and $R^{-1}=800 \mathrm{GeV}$, close to the upper and lower ends respectively, of this range, noting that both of these choices would predict KK excitations which could be accessible at planned $e^{+} e^{-}$ machines. The value of the cutoff scale $\Lambda$ is a somewhat tricky question, as it corresponds to the breakdown of the compactification limit - which is, after all, a gradual process. Once again, we choose two benchmark values $\Lambda=20 R^{-1}$ and $50 R^{-1}$ for our analysis, which correspond to an assumption that the low-energy effective theory will remain valid till a scale of some tens of $\mathrm{TeV}$. As with most cutoff scales, the numerical value chosen is only a ballpark value, but this does not really matter as the dependence of the mass spectrum and couplings on $\Lambda$ is logarithmic, and hence not very sensitive to the precise number chosen. A visual presentation of the parameter space of this UED model may be found in Figure 6 .

\begin{tabular}{lcrrrr}
\hline Benchmark Point: & & $(\mathrm{I})$ & $(\mathrm{II})$ & $(\mathrm{III})$ & $(\mathrm{IV})$ \\
\hline Size Parameter & $R^{-1}$ & 400 & 400 & 800 & 800 \\
Cutoff Scale & $\Lambda R$ & 20 & 50 & 20 & 50 \\
\hline \hline$n=1$ excitations & $\gamma_{1}$ & 401.2 & 401.3 & 800.0 & 799.9 \\
& $E_{1}$ & 404.4 & 405.7 & 808.7 & 811.4 \\
& $L_{1}$ & 412.0 & 415.6 & 823.9 & 831.3 \\
& $W_{1}^{ \pm}$ & 430.8 & 437.6 & 850.4 & 864.2 \\
& $Z_{1}$ & 431.4 & 438.1 & 850.5 & 864.3 \\
\hline$n=2$ excitations & $\gamma_{2}$ & 800.7 & 800.6 & 1599.7 & 1599.5 \\
& $L_{2}$ & 818.4 & 825.7 & 1636.8 & 1651.4 \\
& $Z_{2}$ & 840.2 & 854.1 & 1674.4 & 1702.4 \\
\hline \hline
\end{tabular}

Table 1: Partial mass spectrum of the UED model at the four chosen benchmark points, showing only the lowest-lying states. All numbers are in GeV, except for $\Lambda R$, which is dimensionless.

In Table 1, we list the masses of the KK excitations $(n=1,2)$ relevant for the current study, for the four chosen benchmark points:

$$
\begin{array}{rll}
\text { (I) } & R^{-1}=400 \mathrm{GeV}, & \Lambda=20 R^{-1}=8 \mathrm{TeV} ; \\
\text { (II) } & R^{-1}=400 \mathrm{GeV}, & \Lambda=50 R^{-1}=20 \mathrm{TeV} ; \\
(\mathrm{III}) & R^{-1}=800 \mathrm{GeV}, & \Lambda=20 R^{-1}=16 \mathrm{TeV} ; \\
(\mathrm{IV}) & R^{-1}=800 \mathrm{GeV}, & \Lambda=50 R^{-1}=40 \mathrm{TeV} .
\end{array}
$$

In every case, the $\gamma_{1}$ (partnering the massless photon $\gamma$ ) is the LKP, while the next-tolightest $n=1$ excitation is the $\mathrm{SU}(2)$ singlet lepton $E_{1}$, followed by the $\mathrm{SU}(2)$ doublet leptons $L_{1}=\left(\nu_{1}, \ell_{1}\right)^{T}$. For all practical purposes, the three generations of $E_{1}$ are degenerate, and a similar statement can be made about the $L_{1}$ as well. These states, together with the $W_{1}^{ \pm}$and the $Z_{1}$, all lie in the ballpark (within about $15 \%$ ) of $R^{-1}$ - the splitting mostly coming from radiative corrections. In the $2 R^{-1}$ regime, the relevant particles are $\gamma_{2}, Z_{2}$, 
and the $\mathrm{SU}(2)$ doublet lepton $L_{2}=\left(\nu_{2}, \ell_{2}\right)^{T}$, whose $\mathbb{Z}_{2}$-even components are left-chiral. The singlet $E_{2}$, whose $\mathbb{Z}_{2}$-even component is right-chiral, though having a mass close to $2 R^{-1}$, hardly couples to the $Z_{2}$ because the latter is almost completely dominated by the third component of the $W_{2}$ triplet. Other excitations, e.g. of quarks, are generically heavier than the corresponding ones shown in the table, because they have larger radiative corrections (for quarks, this is mainly due to large colour factors). Since this class of KK excitations is not relevant for our analysis, we do not exhibit the corresponding masses in Table 1 .

At this point it is worth recalling the rule-of-thumb that if there is enough energy to pairproduce $n=1$ excitations, then there is also enough energy to excite a single $n=2$ resonance. Single production of $n=1$ states is, as we have seen, forbidden by the conservation of KK parity. Now, at the ILC-1, running at $\sqrt{s}=500 \mathrm{GeV}$, no $n=1 \mathrm{KK}$ excitation can be pair-produced, in view of the lower limit $R^{-1} \gtrsim 300 \mathrm{GeV}$, neither can the $n=2$ resonances be singly produced. Thus, the first phase of the ILC would be uninteresting for UED searches. At the ILC-2 - the energy upgrade running at $\sqrt{s}=1 \mathrm{TeV}$ - pair production of $n=1$ states as well as single production of $n=2$ resonances can take place if $R^{-1}<500$ $\mathrm{GeV}$. As this certainly holds for our first pair of benchmark points (I) and (II), we focus largely on the ILC-2 for our analysis. To study the heavier states that are predicted for $500 \mathrm{GeV}<R^{-1}<900 \mathrm{GeV}$ - where our second pair of benchmark points (III) and (IV) lie — we shall need the CLIC, running at $\sqrt{s}=3 \mathrm{TeV}$ and $5 \mathrm{TeV}$, with a planned [20] luminosity of $10^{35} \mathrm{~cm}^{-2} \mathrm{~s}^{-1}$. This machine could, in fact, explore the complete $n=1$ spectrum as well as the $n=2$ resonances all the way up to the cosmological upper limit of $R^{-1}$ mentioned above.

Since KK-parity is conserved, the possible decay modes for the low-lying KK states listed in Table 1 are severely restricted. A list of the important decay modes is given below. The decay channels important for this work are marked with a tic $(\sqrt{ }) \operatorname{sign}$.

$$
\begin{aligned}
& \gamma_{1}: \text { stable (invisible) } \\
& E_{1}: E_{1} \rightarrow \ell+\gamma_{1} \\
& \sqrt{ } \quad L_{1}: \nu_{1} \rightarrow \nu+\gamma_{1}, \ell_{1} \rightarrow \ell+\gamma_{1} \\
& W_{1}: W_{1} \rightarrow \nu+\bar{\ell}_{1}, \ell+\bar{\nu}_{1} \\
& \sqrt{ } \quad Z_{1}: Z_{1} \rightarrow \nu+\bar{\nu}_{1}, \ell+\bar{\ell}_{1} \\
& \mid \begin{array}{llll}
\sqrt{ } \quad \gamma_{2} & : & \gamma_{2} \rightarrow f+\bar{f} \\
E_{2} & : & E_{2} \rightarrow \ell+\gamma_{2} \\
L_{2} & : & \nu_{2} \rightarrow \nu_{1}+\gamma_{1}, \ell_{2} \rightarrow \ell_{1}+\gamma_{1} \\
& & \\
\sqrt{ } \quad Z_{2} & : & Z_{2} \rightarrow f+\bar{f}, \ell_{1}+\bar{\ell}_{1}, \nu_{1}+\overline{\nu_{1}}
\end{array}
\end{aligned}
$$

Here, $f$ stands for any generic $n=0$ fermion (leptons as well as quarks) while $\ell$ and $\nu$ stand, respectively, for charged and neutral components of the $\mathrm{SU}(2)$ doublet leptons $L$. The $\mathrm{SU}(2)$ singlet (charged) leptons are denoted $E$. Generation indices have been suppressed and SM particles are written without the $n=0$ subscript. It is important to note that the above list, limited as it is, contains both KK number-conserving as well as KK-number-violating 
processes. A generic feature of the UED model is that the branching ratios for the latter, though nonzero, are suppressed by the boundary-to-bulk ratio. The branching ratios for the KK number-conserving decays are, in turn, suppressed by the limited phase space available, because states with the same KK number have rather small mass-splittings. Eventually, therefore, both types of decay turn out to be of comparable importance. It is important to note that, unlike the $Z$-boson in the $\mathrm{SM}$, the $Z_{1}$ decays overwhelmingly through the leptonic channels, i.e. either $Z_{1} \rightarrow \ell^{+} \ell^{-} \gamma_{1}$, through a resonant $\ell_{1}^{ \pm}$(which decays to $\ell^{ \pm} \gamma_{1}$ with unit branching ratio), or, $Z_{1} \rightarrow \nu \bar{\nu} \gamma_{1}$, through a resonant $\nu_{1}$ (which decays to $\nu \gamma_{1}$, again with unit branching ratio). Since $n=1$ excitations of quarks are generically heavier than the $z_{1}$, its hadronic decays occur through three-body processes, and have partial widths that are at least three orders of magnitude smaller than their leptonic counterparts. Obviously, out of the 6 lepton flavours $\left(e, \mu, \tau\right.$ and $\left.\nu_{e}, \nu_{\mu}, \nu_{\tau}\right)$ available in $Z_{1}$ decays, dilepton plus missing $E_{T}$ signals can be obtained only by counting the $e, \mu$, and $\tau$ flavours. This means that only $50 \%$ of the $Z_{1}$-s produced at an $e^{+} e^{-}$machine will lead to observable signals.

(a) $\gamma_{2}$ with doublet fermions

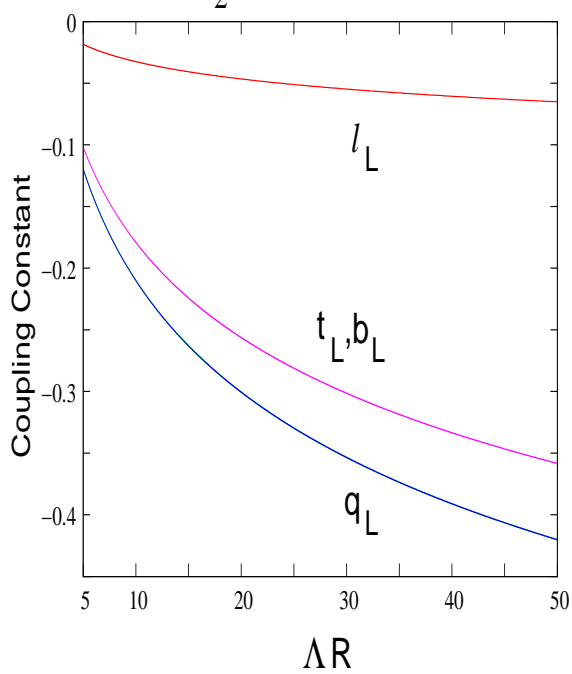

(b) $\gamma_{2}$ with singlet fermions

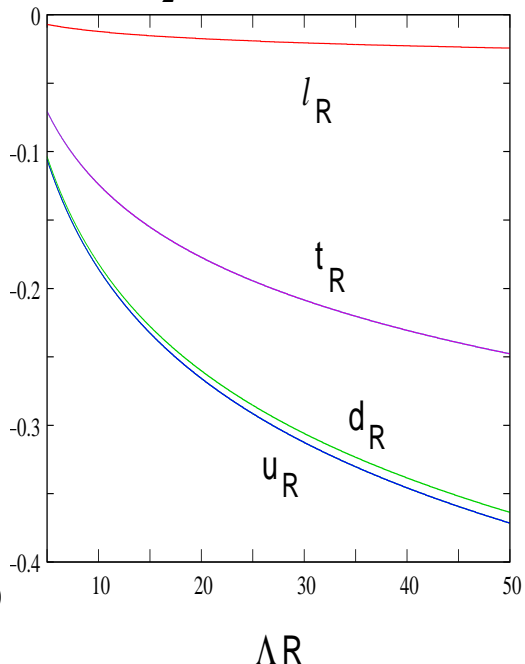

(c) $Z_{2}$ with doublet fermions

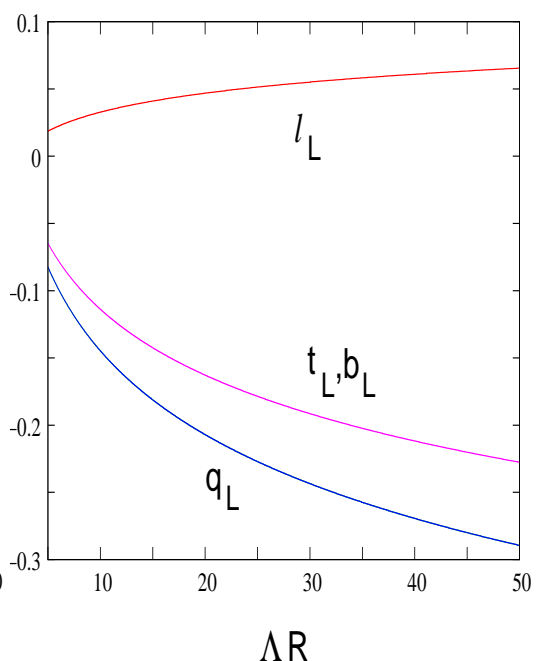

Figure 2: Illustrating the variation in coupling of fermion pairs to the $\gamma_{2}$ and $Z_{2}$ excitations. Each box carries a header explaining the type of coupling, and each curve carries a legend explaining which fermions are involved. In every box, $l_{L, R}$ stands for any charged lepton $e, \mu$ or $\tau$, and $t_{L, R}$ stands for the top quark while $b_{L}$ indicates the bottom quark. In the boxes marked (a) and (c), $q_{L}$ stands for any light quark $u, d, s$ or c. In the box marked (b), $u_{R}$ includes $c_{R}$, while $d_{R}$ includes both $s_{R}$ and $b_{R}$. Note that the $Z_{2} l_{L} \bar{l}_{L}$ coupling is positive, while all others are negative.

To study the production and decay modes of the $\gamma_{2}$ and $Z_{2}$ resonances, we need to know how they couple to the SM $(n=0)$ fermions. As the relevant formulae may be obtained from Ref. [4] or Ref. [9], we have merely illustrated the results here. In Figure 2, we show the variation of the couplings of $n=0$ fermion-antifermion pairs with the $n=2$ electroweak gauge bosons as $\Lambda R$ varies from 5 to 50 . The $\Lambda R$ dependence originates from the fact that 
these couplings conserve KK-parity, but violate KK-number and hence are very sensitive to radiative corrections. A cursory examination of Figure 2 is enough to establish that the magnitudes of these couplings increase more or less as $\log \Lambda$. This is, of course, characteristic of an effective theory and is not incompatible with the Froissart bound. We also note the fact that at the $n=2$ level, the 'Weinberg angle' quantifying the mixing between $W_{2}^{3 \mu}$ and $B_{2}^{\mu}$ states is so small that it is quite reasonable to take the $Z_{2}^{\mu}$ as a pure $W_{2}^{3 \mu}$, coupling only with doublet fermions, at least for all practical purposes. In fact, the tiny $Z_{2} f_{R} \bar{f}_{R}$ couplings hardly play any part in our discussions and hence we do not exhibit them with the others in Figure 2.

It is interesting that the production cross-sections of the $n=2$ resonances are quite viable even though the coupling strength to an $e^{+} e^{-}$pair, as exhibited in Figure 2, is quite small $\left(\sim 10^{-2}\right)$. This happens, of course, because of the resonance effect, where the coupling constant cancels out of the final cross-section in the narrow-width approximation. The resonant cross-section remains viable even when convoluted with the electron luminosity function $f_{e / e}(x)$. Naturally, this grows stronger as the machine energy and the resonant mass approach each other as $x \rightarrow 1$. On the other hand, in the same limit, the cross-section for producing a pair of $n=1$ excitations gets suppressed since the phase space gets squeezed towards zero volume. The phase space suppression factor, roughly $\left(1-4 / R^{2} s\right)^{3 / 2}$ is, in fact, enough to neutralise the larger coupling of $n=1$ states to SM fermions, so that, eventually, the pair-production cross-section becomes somewhat smaller than the dijet cross-section. In addition, when we trigger only on final states with charged leptons, there is an additional suppression factor of $\frac{1}{2} \times \frac{1}{2}$, which reduces the cross-section to a quarter. Kinematic cuts further suppress this cross-section, as explained in Section 4. Despite all these suppression factors, however, the high luminosity expected at the ILC or CLIC serves to keep the $4 \ell+E_{T}$ signal viable. Since this is one of the cleanest signals we can have, with a very small SM background, it is well worth including in our study, as we shall see presently.

In this section, then, we have enlisted the details of the UED model that are relevant for our numerical analysis of the problem and for prediction of the relevant signals. In the next section, we describe how to include radiation effects and obtain an effective spectrum which can be convoluted with the formulae in Ref. [9] to get realistic predictions, using the choices of free parameters delineated in the present section.

\section{$3 \quad$ ISR and Beamstrahlung}

To study the effect of ISR and beamstrahlung in electron (positron) beams, we make use of the so-called structure function formalism [21]. Assuming that an initial electron (positron) 
of energy $E_{b}$ emits a photon of energy $E_{\gamma}$, leaving an electron (positron) of energy $E_{e}$, we define the energy fraction $x=E_{e} / E_{b}$ so that $E_{\gamma}=(1-x) E_{b}$. We now define a normalised probability distribution $f_{e / e}(x)$ for the colliding electron (positron) to have energy $E_{e}=x E_{b}$. This quantity is analogous to the parton density function $f_{q / p}(x)$ or $f_{g / p}(x)$ at a hadron collider. Hence, if we consider the process $e^{+}\left(p_{1}\right) e^{-}\left(p_{2}\right) \rightarrow q \bar{q}(\gamma)$, the cross-section will be

$$
\sigma\left[e^{+}\left(p_{1}\right) e^{-}\left(p_{2}\right) \rightarrow q \bar{q}(\gamma)\right]=\int d x_{1} d x_{2} f_{e / e}\left(x_{1}\right) f_{e / e}\left(x_{2}\right) \quad \hat{\sigma}\left[e^{+}\left(x_{1} p_{1}\right) e^{-}\left(x_{2} p_{2}\right) \rightarrow q \bar{q}\right]
$$

where $\hat{\sigma}$ denotes the cross-section calculated with the (degraded) initial momenta $x_{1} p_{1}$ and $x_{2} p_{2}$. The effective centre-of-mass energy is $\sqrt{\hat{s}} \approx \sqrt{x_{1} x_{2} s}$, where $s=\left(p_{1}+p_{2}\right)^{2}$ and $\hat{s}=$ $\left(x_{1} p_{1}+x_{2} p_{2}\right)^{2}$ in the high energy limit when the electron (positron) mass can be neglected.

The electron 'luminosity' function $f_{e / e}(x)$ is a combination of the probabilities for both ISR and beamstrahlung effects and it may be calculated [21] by convoluting the corresponding (normalised) spectral densities as follows:

$$
f_{e / e}(x)=\int_{x}^{1} \frac{d y}{y} f_{e / e}^{\mathrm{ISR}}(y) f_{e / e}^{\mathrm{beam}}\left(\frac{x}{y}\right)
$$

For the ISR spectral density $f_{e / e}^{\mathrm{ISR}}(y)$, we use a one-loop corrected Weizäcker-Williams approximation to write

$$
f_{e / e}^{\mathrm{ISR}}(y)=\frac{\omega}{16}\left[(8+3 \omega)(1-y)^{\omega / 2-1}-4(1+y)\right]
$$

where

$$
\omega=\frac{2 \alpha}{\pi}\left(\log \frac{s}{m_{e}^{2}}-1\right)
$$

with $\alpha$ and $m_{e}$ denoting the fine-structure constant and the electron mass respectively, both evaluated at the scale $E_{b}$. Because of its weak logarithmic dependence on $s, \omega$ stays confined in a small range around $0.14 \pm 0.02$ for the energies under consideration. As a result the exponent in Eq. (4) is always negative, indicating that there will be a steep rise in $f_{e / e}^{\mathrm{ISR}}(y)$ as $y \rightarrow 1$. The normalisation condition $\int_{0}^{1} d y f_{e / e}^{\mathrm{ISR}}(y)=1$ ensures that there is no singularity, but the large values near $y=1$ indicate that ISR effects are not very effective in spreading out the energy $E_{e}$ much below $E_{b}$.

The formula for the spectral density due to beamstrahlung is much more complicated, as it depends critically on the (dimensionless) beamstrahlung parameter $\Upsilon$. This is given, for $e^{-}\left(e^{+}\right)$beams with a Gaussian energy profile, by [22]

$$
\Upsilon=\frac{5 r_{e}^{2}}{6 \alpha m_{e}} \frac{E_{b} N_{e}}{\sigma_{z}\left(\sigma_{x}+\sigma_{y}\right)}
$$


where $r_{e} \approx 2.8 \times 10^{-15} \mathrm{~m}$ is the classical electron radius, $\sigma_{x, y, z}$ are the dimensions of a bunch (assuming an ellipsoidal shape) and $N_{e}$ is the number of electrons in a bunch. The spectral density for multiple photon emission can be approximated [22], for values of $\Upsilon$ less than about 10, by the formula

$$
f_{e / e}^{\text {beam }}(\xi)=\frac{1}{N_{\gamma}}\left[\delta(1-\xi)\left(1-e^{-N_{\gamma}}\right)+\left(1-\xi+\xi \sqrt{1+\Upsilon^{2 / 3}}\right) \frac{e^{-\eta(\xi)}}{1-\xi} \sum_{r=0}^{\infty} \frac{\eta(\xi)^{r / 3} \Gamma_{r+1}\left(N_{\gamma}\right)}{r ! \Gamma\left(\frac{r}{3}\right)}\right]
$$

where

$$
N_{\gamma}=\frac{5 \alpha^{2} m_{e} \sigma_{z}}{2 r_{e} E_{b}} \frac{\Upsilon}{\sqrt{1+\Upsilon^{2 / 3}}}
$$

is the number of photons emitted per electron and $\eta(\xi)=2(1-\xi) / 2 \Upsilon \xi$. The symbol $\Gamma(x)$ stands for the usual Euler gamma function while $\Gamma_{s}(x)$ denotes the incomplete gamma function defined by

$$
\Gamma_{s}(x)=\int_{0}^{s} d t t^{1+x} e^{-t}
$$

This formula is too complicated to reveal much to an inspection except, clearly, the important role played by the $\Upsilon$ parameter, but, when plotted as a function of the argument $\xi$, it does have a shape rather similar to that of the ISR spectrum - except that the peak around $\xi=1$ is not quite so sharp. However, what matters for our analysis is neither the ISR spectrum $f_{e / e}^{\mathrm{ISR}}(y)$ alone, nor the beamstrahlung function $f_{e / e}^{\text {beam }}(\xi)$, but the convolution of the two shown in Equation (3), which will describe the actual energy spread. Some of the important parameters required to generate these spectra are given below, in Table 2 .

\begin{tabular}{ccccc}
\hline Machine & $\sqrt{s}(\mathrm{TeV})$ & $\sigma_{z}(\mathrm{~mm})$ & $\omega$ & $\Upsilon$ \\
\hline \hline ILC-1 & 0.5 & 0.30 & 0.1235 & 0.048 \\
ILC-2 & 1.0 & 0.30 & 0.1300 & 0.110 \\
CLIC & 3.0 & 0.03 & 0.1402 & 8.100 \\
\hline \hline
\end{tabular}

Table 2: Some of the beam parameters for the ILC and CLIC which are crucial to the evaluation of radiation spectra involving ISR and beamstrahlung.

In Figure 3 we have shown the behaviour of this 'luminosity' function as the momentum fraction $x$ of the colliding electron (positron) varies from 0 to 1 . The solid (red, blue) lines show the behaviour at the ILC-1 and ILC-2, running at $\sqrt{s}=500 \mathrm{GeV}$ and $\sqrt{s}=1 \mathrm{TeV}$ respectively, while the dashed (green) line shows the corresponding curve at the CLIC, running at $3 \mathrm{TeV}$. The dotted (black) line shows the ISR spectrum at the ILC-2 and is given essentially for purposes of comparison.

The above graph allows one to make some quick estimates of the size of the resonance effects that are induced by radiative returns. For example, if the resonance occurs when the energy 


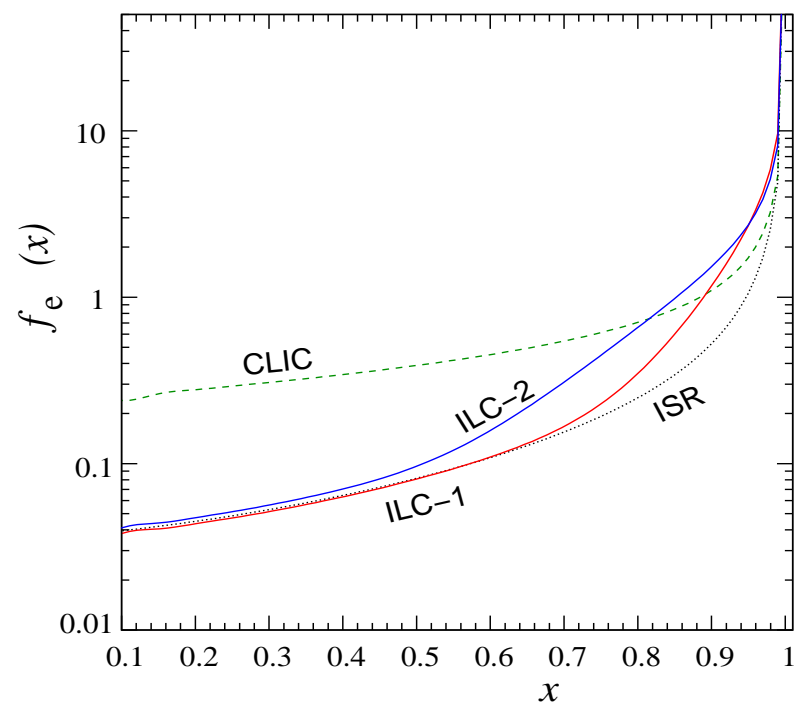

Figure 3: Effective electron (positron) flux at a high energy collider taking into account both ISR and beamstrahlung effects. Note the steep rise as $x \rightarrow 1$ and also the fact that the spreading effect is much larger at the higher energy of CLIC.

of the colliding particles is around $60 \%$ of the machine energy, then we may expect $x \simeq 0.75$, where the luminosity at the ILC is around $1 \%$ of the value expected in the vicinity of $x \rightarrow 1$. This means that for both $e^{+}$and $e^{-}$combined, we get an effective flux of the order of $10^{-4}$. For reasonably sharp resonances, this small flux, compounded with the typical resonant cross-section, which is at least $10^{4}$ times the off-resonance cross-section (as the decay widths of $\gamma_{2}$ and $Z_{2}$ are in the ballpark of $1 \mathrm{GeV}$ ), would predict at least as large a contribution as that which we would predict in the absence of the radiative effects. For relatively lighter resonances, the effect is more pronounced at the CLIC, as Figure 3 readily shows. Even apart from the contribution to the total cross-section, which is already considerable, we will get a more dramatic effect if we look at the invariant mass distribution of the particles produced through the resonant state. The next section discusses this issue in more detail.

\section{Bump Hunting}

In order to make a numerical analysis and illustrate the effect of radiative returns, we have incorporated the electron luminosity function of Eq. (3) in a simple Monte Carlo event generator where we calculate

(a) the dijet production process $e^{+}+e^{-} \rightarrow \gamma^{*} / Z / \gamma_{2} / Z_{2} \rightarrow q+\bar{q}$, and

(b) the pair-production process $e^{+}+e^{-} \rightarrow Z_{1}+Z_{1} \rightarrow \gamma_{1} \ell^{+} \ell^{-}+\gamma_{1} \ell^{+} \ell^{-}$,

which have been illustrated in Figure 1. In case (a), the final state will be a pair of jets, and we focus on the invariant mass distribution of these jets, where distinct peaks corresponding 
to resonant $\gamma_{2}$ and $Z_{2}$ states should be seen over the SM background arising from $\gamma^{*}$ and $Z^{*}$ exchanges. In case $(b)$, the final state will be $\ell^{+} \ell^{+} \ell^{-} \ell^{-}$and a large missing $E_{T}$, where the four leptons can be either electrons (positrons), muons (anti-muons) or taus (anti-taus). As a check, we have also incorporated the UED fields and couplings as a separate kernel in the software CalcHEP [23] and generated the same signals, using the ISR and beamstrahlung generators built into this software. In every case, the numbers obtained from CalcHEP turn out to be in very close agreement with those obtained from our simulation codes.

As discussed before, we do not consider the ILC-1 for this analysis, because mass scales as large as $2 \times 400 \mathrm{GeV}$ are not kinematically accessible at a $500 \mathrm{GeV}$ machine. Our results for the distribution in dijet invariant mass at the ILC-2 $(\sqrt{s}=1 \mathrm{TeV})$ are illustrated in Figure 4 . For these plots, we have chosen the benchmark points (I) and (II) with $R^{-1}=400 \mathrm{GeV}$ and $\Lambda R=20$ and 50 respectively, where the $\gamma_{2}$ and $Z_{2}$ resonances are light enough to be produced on-shell. We plot the invariant mass distribution as a histogram with bin-size 20 $\mathrm{GeV}$. This bin width is dictated by a simple-minded estimate of the detector-smearing effects on the measurement of the jet momentum. As these measurements will be calorimetric, the error is dominated by the error in the energy measurement, which is estimated [24] as $\delta E_{J} \approx \pm 0.3 \sqrt{E_{J}}$. Considering two jets of the same energy with errors adding in quadrature 6 , the error in dijet invariant mass arising from energy measurements alone can be estimated as $\delta M_{J J} \approx \sqrt{2} \times \delta E_{J} \approx \pm 0.42 \sqrt{E_{J}}$. For the ILC-2, running at $\sqrt{s}=1 \mathrm{TeV}, E_{J} \simeq 500 \mathrm{GeV}$, which means that $\delta M_{J J} \approx \pm 9.4 \mathrm{GeV}$. If we add on the smaller errors due to thrust axis and angle measurements, we can expect an error of around $\delta M_{J J} \approx \pm 10 \mathrm{GeV}$. Thus, it is reasonable to choose a bin width of $20 \mathrm{GeV}$.

To identify the jets, we impose the following acceptance cuts:

- The pseudo-rapidity $\eta_{J}$ of the jets should satisfy $\left|\eta_{J}\right| \leq 2.5$;

- The jet transverse momentum $p_{T}^{(J)}$ should satisfy $p_{T}^{(J)} \geq 10 \mathrm{GeV}$.

These represent minimal requirements for identification of jets in the detectors that would be built to operate at ILC energies and means that we are considering essentially the entire phase space available in practice. We also require, however, to ensure that the dijets triggered on are being produced strictly from beam-beam collisions, and for this, we impose a selection cut:

- The missing transverse momentum $\not \not_{T}$ should satisfy $\not \not_{T} \leq 10 \mathrm{GeV}$.

\footnotetext{
${ }^{6}$ This is consistent with the construction of the invariant mass in terms of the momenta (energies).
} 
The choice of $10 \mathrm{GeV}$ for these selection and acceptance cuts on the jets is consistent with the uncertainty in jet energy described above.

Since this work is essentially of exploratory nature, our event generator works only at the parton level, i.e. we identify the jet thrust axis and the jet energy-momentum with the direction and magnitude of the four-momentum of the parent quark. No simulation of the fragmentation of these quarks is attempted. For an $e^{+} e^{-}$collider, this is known to be a reasonably good approximation when averaged over a large number of events, though an individual event may occasionally have very different characteristics. The analysis may, therefore, be carried out fairly accurately without using more sophisticated jet simulation algorithms, provided there is enough luminosity to yield a large number of events. The advantage of using a fast parton-level code is that it readily allows exploration of the parameter space beyond the four benchmark points chosen earlier. This advantage makes itself felt in the last section, where we discuss the entire accessible parameter space rather than a few points.
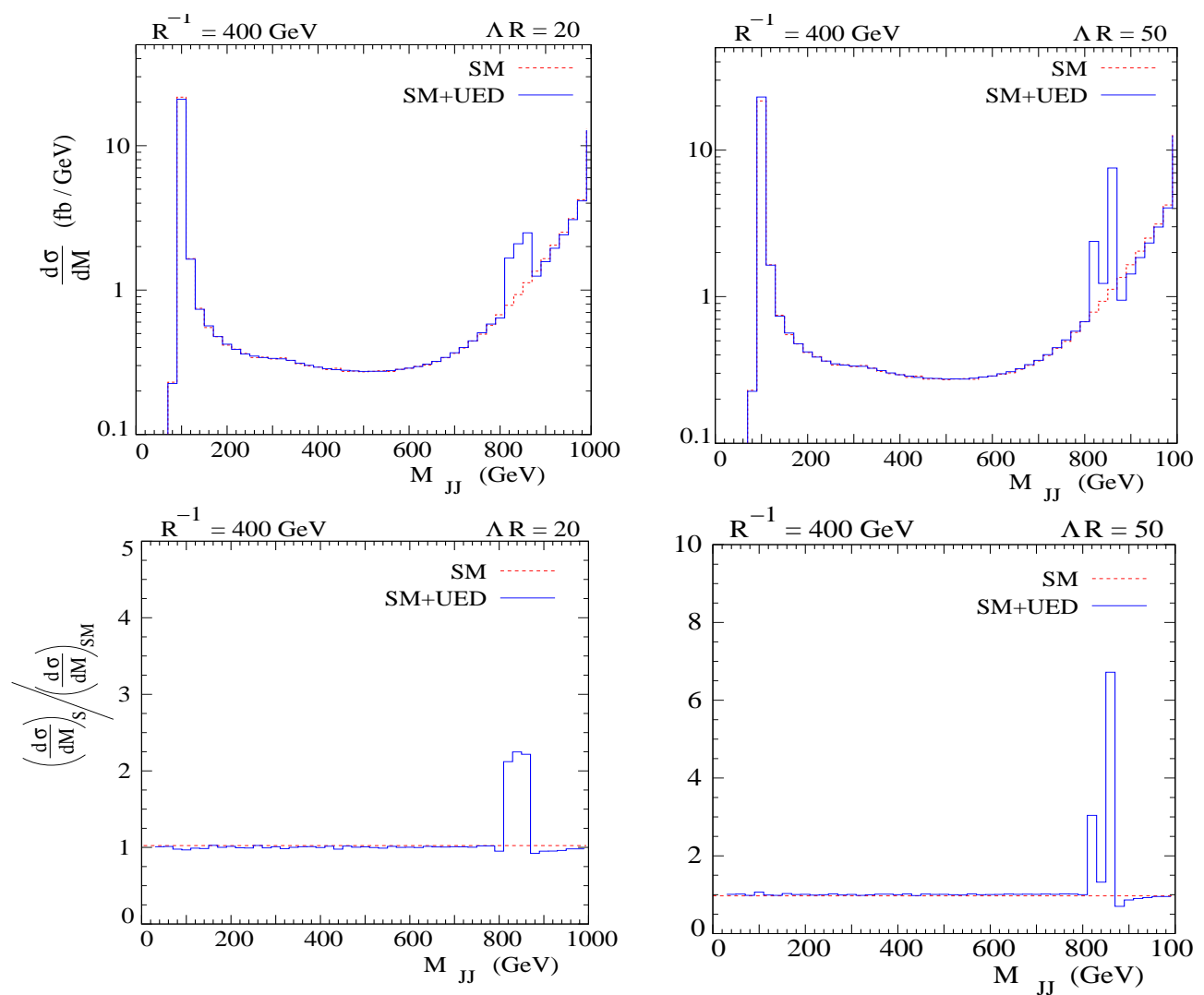

Figure 4: Illustrating $\gamma_{2}$ and $Z_{2}$ resonances at the ILC-2, running at $\sqrt{s}=1 \mathrm{TeV}$. In the upper boxes, the dashed (red) histograms correspond to the SM prediction, while the solid (blue) histograms represent the effect of UED signals for $R^{-1}=400 \mathrm{GeV}$ and $\Lambda R=20$ (left) and 50 (right). In the lower boxes, the same cross-sections are normalised by the SM prediction, effectively throwing the new physics effect into relief. 
Our results for the invariant mass distribution are exhibited in Figure 4. The upper and lower boxes on the left show the distribution in invariant mass of the dijet in the final state for the benchmark points (I) and (II), i.e. $\Lambda R=20$ and 50, respectively. The solid (blue) histogram shows the effect of the UED signal added to the SM background, while the dashed (red) histogram - just about visible below the peak(s) - represents the SM alone. As expected, the overall shape of the SM histogram reproduces the luminosity curve 7 in Figure 3 , with the $x \rightarrow 1$ peak corresponding to the machine energy of $1 \mathrm{TeV}$. Instead of falling off at low $\sqrt{s}$ values, i.e. low $x$, however, the curve rises to a sharp peak in the bin $M_{J J}=90-110 \mathrm{GeV}$, which represents a 'return' to the $Z$-resonance, similar to what was observed at the LEP-1.5. The resonance is nowhere near as strong as it was at LEP-1.5 because the electron flux, as shown in Figure 3 is quite low (around $4 \%$ ) at $x=M_{Z} / \sqrt{s} \simeq 0.09$. At the extreme left end, the cross-section may be seen to drop rather abruptly towards zero - this is not a dynamical effect, but an artefact of the acceptance cuts on the jets.

The (blue) signal histograms in Figure 4, i.e. when the effects of $n=2$ resonances are included, correspond closely to the SM for most of the range in $M_{J J}$, except for the resonant peak(s) around $800-850 \mathrm{GeV}$, which represent(s) on-shell production of the $\gamma_{2}$ and $Z_{2}$ modes. If $\Lambda R=20$, the two peaks, which are separated by around $40-50 \mathrm{GeV}$ at the centres (see Table 1), cannot be resolved with a realistic binning in the invariant mass spectrum, but if $\Lambda R=50$, we obtain two distinct bumps. These are highlighted in the lower boxes, which plot the ratio of the signal histogram to the background histogram. The ratio is unity for most of the invariant mass range, except at the resonant peaks, where the excess stands out decisively. Small dips in the signal below the SM background, just observable to the right of the peaks, correspond to interference effects.

The following points may now be noted in the context of these resonances.

1. The $\gamma_{2}$ and $Z_{2}$ resonances are narrow $(\Gamma \sim 1 \mathrm{GeV})$ and sharp 8 , corresponding to at least a twofold increase in the cross-section in the relevant bin(s), even when multiplied by the small flux factor. Note that the upper left box in Figure 4 shows a single smeared-out resonance because the illustrated bump actually contains two unresolved resonances. As a matter of fact, we have checked that a finer binning of $10 \mathrm{GeV}$, were it experimentally possible, would have been enough to resolve the $\gamma_{2}$ and $Z_{2}$ peaks clearly even for $\Lambda R=20$. Unfortunately, as we have discussed above, this would not be achievable at the ILC for $\sqrt{s}=1 \mathrm{TeV}$.

\footnotetext{
${ }^{7}$ Strictly speaking, the convolution of two such luminosity curves - one for the $e^{-}$and one for the $e^{+}$

${ }^{8}$ The actual decay widths for the $\gamma_{2}\left(Z_{2}\right)$ in $\mathrm{GeV}$ are 0.23 (0.51), 0.45 (0.98), $0.46(0.98)$ and 0.90 (1.91) for the benchmark points I, II, III and IV respectively.
} 
2. Since the excess appears only in one or two bins - and even then the peaks are not very high - the total dijet cross section will show a rather modest deviation from the SM. In order to reduce the SM background, therefore, we may impose some further selection cuts. A glance at Figure 4 shows that a cut of $M_{J J}>600 \mathrm{GeV}$ would effectively reduce the SM background, without in the least affecting the resonances appearing in the 800-900 GeV ballpark. Such a cut is called for anyway, since we know that $R^{-1}>300 \mathrm{GeV}$, i.e. no resonances are expected below $2 \times 300 \mathrm{GeV}$. Similarly, a cut on $p_{T}^{\text {jet }}>100 \mathrm{GeV}$ would remove all vestiges of the Jacobian peak arising from $Z$-decay, without significantly affecting the Jacobian peaks from the decays of the much heavier $\gamma_{2}$ and $Z_{2}$, even when we take smearing effects (due to spread in beam energy) into account. These selection cuts are not needed to discover resonances, but will be needed for the studies of the next section, where we show how the underlying model can be identified unambiguously.

3. Though we have not considered $e^{+} e^{-} \rightarrow \ell^{+} \ell^{-}$processes in this work because of the small branching ratios of the $\gamma_{2}$ and $Z_{2}$ to leptonic channels, it may still be worth considering these channels, since the lepton momenta can be measured much more accurately, allowing for a finer binning of $\ell^{+} \ell^{-}$invariant mass, and hence a clear resolution between the two resonant peaks for all values of $\Lambda R$. If, indeed, a clear signal of the kind predicted in this work is observed, then the resolution of the single peak into two closely-separated peaks might serve to clinch the issue of whether the underlying model incorporates UED or not. However, as the cross-section is small for $\ell^{+} \ell^{-}$final states, such a result will be possible only with a large amount of statistics, and it may take all the data accumulated in the full run of the ILC-2 before we can reach any such conclusion. For this reason, we do not make any analysis of the $\ell^{+} \ell^{-}$ final states in this work.

We have already remarked that even when the $\gamma_{2}, Z_{2}$ resonances in Figure 4 add up to a single bump, the height of this is rather moderate, as resonances go. The situation is significantly improved if we go from the $1 \mathrm{TeV}$ ILC-2 to the $3 \mathrm{TeV}$ CLIC, where the large design value of $\Upsilon$ would be responsible for creating a much wider energy spread. This is illustrated in Figure 5, where the notations and conventions of Figure 4 are repeated. To avoid losing out too heavily on the low flux at low $x \approx 2 R^{-1} / \sqrt{s}$, as well as to illustrate the superior kinematic reach of this higher energy machine, the value of $R^{-1}$ has been chosen as $800 \mathrm{GeV}$, rather than $400 \mathrm{GeV}$, for this figure, pushing the resonance energy to the neighbourhood of 1.6-1.7 TeV. The bin-width has been increased to $30 \mathrm{GeV}$, which is compatible with jets of energy $1.5 \mathrm{TeV}$ each and errors in energy measurement similar to those at the ILC. A comparison of the the two figures will immediately show that at the CLIC, the resonant effect is clearer than at the ILC, the bump rising two to three times higher than the SM 

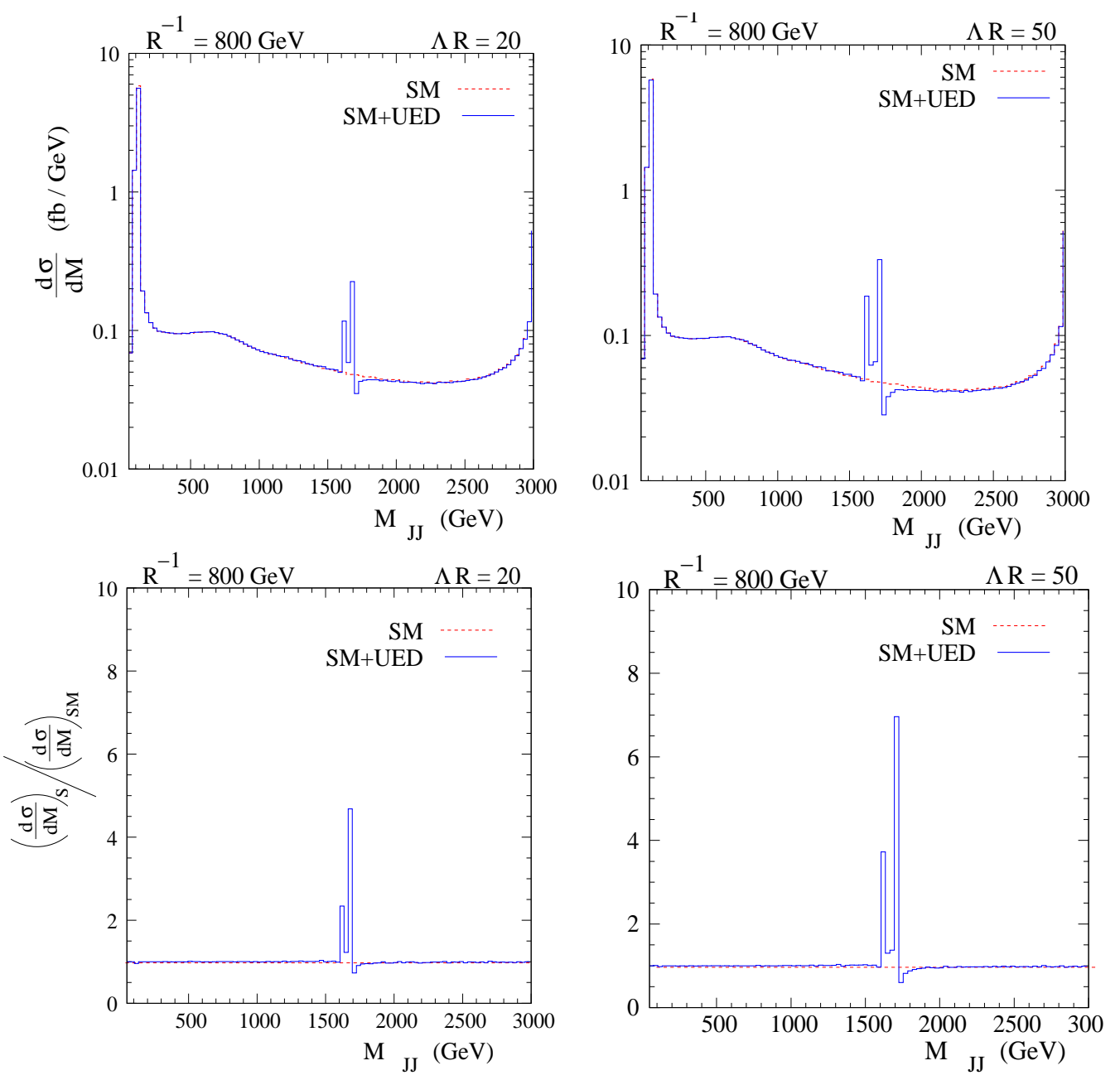

Figure 5: Illustrating $\gamma_{2}$ and $Z_{2}$ resonances at the CLIC, running at $\sqrt{s}=3 \mathrm{TeV}$. All conventions are the same as in Figure 4, except that the parameter $R^{-1}$ is chosen to be $800 \mathrm{GeV}$ and the bins are each $30 \mathrm{GeV}$ wide.

background in the most relevant bin. This greater height is due to a combination of three effects, viz. (i) the greater energy spread due to beamstrahlung at the CLIC (see Fig. 3), (ii) the larger $s$-channel suppression for the background at the higher energy of the CLIC, and (iii) the greater bin size. It is worth noting that at higher values of $R^{-1}$, such as have been chosen for Figure 5, the separation between the $\gamma_{2}$ and $Z_{2}$ peaks is large enough to be clearly distinguishable, in spite of the increased bin size. The fact that the resonance(s) occur in the middle of the available range for $M_{J J}$ also shows that the CLIC will have ample energy to scan the entire range of $R^{-1}$ which is permitted by the dark matter constraint.

We reiterate the fact that a $Z_{1}$ pair and a single $Z_{2}$ (or $\gamma_{2}$ ) are kinematically accessible for the same machine energy, indicating that there will always be a simultaneous excess over the SM prediction in the cross-sections for $e^{+} e^{-} \rightarrow$ dijets, as well as in $e^{+} e^{-} \rightarrow 4 \ell+E_{T}$. 
The presence of both these excess contributions would constitute a strong signal for new physics of the type considered in this work. Production and decay of $Z_{1}$ pairs at an $e^{+} e^{-}$ collider has been discussed in the literature [25], and hence we do not elaborate on the signal characteristics in this paper. As explained in a previous section, we concentrate on the dominant leptonic decay modes of the $Z_{1}$, viz.

$$
Z_{1} \rightarrow \ell+\bar{\ell}_{1} \rightarrow \ell+\left(\bar{\ell}+\gamma_{1}\right)
$$

leading to a $4 \ell+E_{T}$ signal at the ILC or CLIC. This can take place in two ways: $Z_{1} \rightarrow$ $\ell \bar{\ell}_{1} \rightarrow \ell\left(\bar{\ell} \gamma_{1}\right)$ and its charge-conjugate process $Z_{1} \rightarrow \bar{\ell} \ell_{1} \rightarrow \bar{\ell}\left(\ell \gamma_{1}\right)$, both leading to the same final state $\ell \bar{\ell}+\not_{T}$. The branching ratios are the same for all lepton flavours in the massless limit. Since there are six leptons $\left(\ell=e, \mu, \tau, \nu_{e}, \nu_{\mu}, \nu_{\tau}\right)$, the branching ratio for this process to each individual flavor must be $\frac{1}{6}$. These branching ratios require to be convoluted with the detection efficiencies to get a more realistic estimate. As stated before, we are interested only in the charged leptons $e, \mu$ and $\tau$. The efficiency factors may safely be taken [24] as 95\% for the $e$ and $\mu$, and $85 \%$ for the $\tau$. Armed with this information, we can analyse the different possibilities for a $4 \ell+\#_{T}$ final state as shown in Table 3 .

\begin{tabular}{cccccl}
\hline Channel & $Z_{1} \rightarrow$ & $Z_{1} \rightarrow$ & Final State & B.R. & Efficiency Factor \\
\hline \hline 1 & $e^{+} e^{-}+E_{T}$ & $e^{+} e^{-}+E_{T}$ & $e^{+} e^{+} e^{-} e^{-}+E_{T}$ & $\frac{1}{6} \times \frac{1}{6}$ & $\times(0.95)^{4}$ \\
2 & $e^{+} e^{-}+E_{T}$ & $\mu^{+} \mu^{-}+E_{T}$ & $e^{+} e^{-} \mu^{+} \mu^{-}+E_{T}$ & $\frac{1}{6} \times \frac{1}{6}$ & $\times(0.95)^{4}$ \\
3 & $e^{+} e^{-}+E_{T}$ & $\tau^{+} \tau^{-}+E_{T}$ & $e^{+} e^{-} \tau^{+} \tau^{-}+E_{T}$ & $\frac{1}{6} \times \frac{1}{6}$ & $\times(0.95)^{2} \times(0.85)^{2}$ \\
\hline 3 & $\mu^{+} \mu^{-}+E_{T}$ & $e^{+} e^{-}+E_{T}$ & $e^{+} e^{-} \mu^{+} \mu^{-}+E_{T}$ & $\frac{1}{6} \times \frac{1}{6}$ & $\times(0.95)^{4}$ \\
4 & $\mu^{+} \mu^{-}+E_{T}$ & $\mu^{+} \mu^{-}+E_{T}$ & $\mu^{+} \mu^{+} \mu^{-} \mu^{-}+E_{T}$ & $\frac{1}{6} \times \frac{1}{6}$ & $\times(0.95)^{4}$ \\
5 & $\mu^{+} \mu^{-}+E_{T}$ & $\tau^{+} \tau^{-}+E_{T}$ & $\mu^{+} \mu^{-} \tau^{+} \tau^{-}+E_{T}$ & $\frac{1}{6} \times \frac{1}{6}$ & $\times(0.95)^{2} \times(0.85)^{2}$ \\
\hline 6 & $\tau^{+} \tau^{-}+E_{T}$ & $e^{+} e^{-}+E_{T}$ & $e^{+} e^{-} \tau^{+} \tau^{-}+E_{T}$ & $\frac{1}{6} \times \frac{1}{6}$ & $\times(0.95)^{2} \times(0.85)^{2}$ \\
7 & $\tau^{+} \tau^{-}+E_{T}$ & $\mu^{+} \mu^{-}+E_{T}$ & $\mu^{+} \mu^{+} \tau^{-} \tau^{-}+E_{T}$ & $\frac{1}{6} \times \frac{1}{6}$ & $\times(0.95)^{2} \times(0.85)^{2}$ \\
8 & $\tau^{+} \tau^{-}+E_{T}$ & $\tau^{+} \tau^{-}+E_{T}$ & $\tau^{+} \tau^{+} \tau^{-} \tau^{-}+E_{T}$ & $\frac{1}{6} \times \frac{1}{6}$ & $\times(0.85)^{4}$ \\
\hline \hline
\end{tabular}

Table 3: Branching ratio (B.R.) and detector efficiency factors for different channels in $Z_{1}$ pair decay.

Multiplying out the factors in every row and adding up all the rows leads to an overall suppression factor of about 0.17 , instead of the $25 \%$ we would have got if we had taken all the efficiencies to be unity.

Finally, before we move on to a more general discussion, we need to note a couple of technicalities in the calculation of these excess contributions, viz.

- For the dijet cross-section, we should look for an identifiable resonance, such as the ones shown in Figures 4 and 5. If the resonance effect is not apparent on inspection - 
as it is in these figures - some numerical criterion may be used instead, such as a $3 \sigma$ deviation from the SM prediction in a pair of adjacent bins. It must be said, however, that within the minimal UED formalism, we will generally have very clear resonances, unless, for some reason, we have to take a very broad bin size.

- For the $4 \ell+\mathbb{E}_{T}$ signal, we should expect the leptons to be relatively soft, since the mass gap between the parent $Z_{1}$ and the daughter $\gamma_{1}$ is not very large. A glance at Table 1 shows that at least for the four benchmark points, this splitting is never more than $35 \mathrm{GeV}$. The analogue of this may not be true for other kinds of new physics which also lead to a $4 \ell+\mathbb{E}_{T}$ final state. Hence, it is reasonable to impose a selection cut $p_{T}^{\ell} \leq 40 \mathrm{GeV}$ on the lepton transverse momentum at the ILC-2, where the parent $Z_{1}$-s are produced with very low $p_{T}$. At the CLIC, however, we will require to raise this cut, since now the $Z_{1}$-s will themselves carry considerable $p_{T}$. We find that a cut of $p_{T}^{\ell} \leq 100 \mathrm{GeV}$ is optimal at the CLIC, as it will hardly affect the signal in a UED model, but can affect the signal in other new physics models quite dramatically.

- For the $4 \ell+E_{T}$ signal, again, one must impose an acceptance cut of $p_{T}^{\ell} \geq 5 \mathrm{GeV}$ $(10 \mathrm{GeV})$ at the ILC (CLIC), since the detectors will obviously not respond to very soft leptons. Unlike the upper cut, this will lead to a significant reduction in the signal cross-section, as the splitting between $n=1$ mass states can sometimes be rather low $(\sim 5-10 \mathrm{GeV})$. However, as this cut is a requirement originating from the hardware constraints, we must perforce live with the corresponding loss in signal events.

The numerical analysis in the following section has been done incorporating these selection criteria. We shall presently see their efficacy as a device to eliminate not merely the Standard Model backgrounds, but also the so-called 'new physics backgrounds' at the ILC.

\section{Tackling the Inverse Problem}

We now address the culminating issue, which may be framed as a question:

Would the observation of a high-mass resonance, or a pair of such resonances, at a high energy $e^{+} e^{-}$collider tell us clearly that a universal extra dimension exists?

This is a classic example of the so-called 'inverse problem' at any high energy machine, i.e. the process of identifying a new physics effect as due to a specific model and a particular set of value of the model parameters. For the case in hand, the answer to the above question

appears to be negative, since it can easily be argued that neither the discovery of resonant peak(s) in the $e^{+} e^{-} \rightarrow J J$ invariant mass spectrum, nor the observation of an excess in 
$4 \ell+\mathbb{E}_{T}$ events, is by itself sufficiently distinctive to be touted as a 'smoking gun' signal of UED. For example, a single bump in the dijet invariant mass spectrum could be interpreted as any one of the following:

1. A resonant $Z^{\prime}$ boson, predicted in four-dimensional models with extra $U(1)$ symmetries.

2. A heavy sneutrino $\widetilde{\nu}_{\mu}$ or $\widetilde{\nu}_{\tau}$ in a SUSY model with $R$-parity-violating couplings of both $L L \bar{E}$ - and $L Q \bar{D}$-type.

3. A massive graviton $G_{1}$, predicted in the Randall-Sundrum model with a warped extra dimension and two $D_{3}$-branes.

If two resonances are clearly resolvable, the possibility of this signal being due to the massive Kaluza-Klein excitation $G_{1}$ of a graviton is more-or-less ruled out. However, one could very well have two nearly-degenerate $Z^{\prime}, Z^{\prime \prime}$ bosons in a model with two extra $U(1)$ symmetries. Similarly, one could have nearly-degenerate sneutrinos, $\widetilde{\nu}_{\mu}$ and $\widetilde{\nu}_{\tau}$ coupling to both $e^{+} e^{-}$ pairs as well as quarks with $R$-parity-violating couplings of similar strength. Of course, it may be possible to identify the spin of the exchanged particle [10] by reconstructing the angular distribution of the jets in the centre-of-mass frame, though this would be hampered by the missing longitudinal momentum due to the radiated photon(s). If this difficulty can be overcome and the angular distribution indicates an exchanged particle of spin 1, the sneutrino and graviton options would be eliminated, but we would still have the $Z^{\prime}$ option to reckon with.

If we consider the $4 \ell+\mathbb{E}_{T}$ signal, standing alone, this can also have many new physics sources. The SM background arises primarily from final states with on-shell production of weak gauge bosons, such as $e^{+} e^{-} \rightarrow W^{+} W^{-} Z \rightarrow\left(\ell^{+} \nu\right)\left(\ell^{-} \bar{\nu}\right)\left(\ell^{+} \ell^{-}\right)$, or from $e^{+} e^{-} \rightarrow$ $Z Z Z \rightarrow\left(\ell^{+} \ell^{-}\right)\left(\ell^{+} \ell^{-}\right)(\nu \bar{\nu})$, where, in principle, each boson can decay into leptons of different flavour. There will also be a large number of sub-dominant diagrams contributing to the same six-lepton final states. As all of these can be accurately predicted in the SM, however, an excess in $4 \ell+\mathbb{E}_{T}$ events would be readily identified. Once such an excess is identified, however, it could be interpreted as due to any of the following alternatives, apart from a UED model.

1. A pair of heavy $Z^{\prime}$ bosons, with an ordinary $Z$ boson radiated from any of the fermion legs, i.e. the process $e^{+} e^{-} \rightarrow Z^{\prime} Z^{\prime} Z$. We would require two of these neutral bosons to decay into $\ell^{+} \ell^{-}$pairs, while the third decays invisibly to neutrinos. Generally, leptons coming from the decay of a $Z^{\prime}$ boson would tend to be hard, and would fall foul of the $p_{T}^{\ell} \leq 40$ (100) GeV cut imposed at the ILC-2 (CLIC). If we further focus on the fact that the $\ell^{+} \ell^{-}$invariant mass will peak at the mass of the parent boson - unlike 
the case for a $Z_{1}$ decay in the UED model, where the leptons are part of a three-body decay - it may be possible to distinguish this case from the UED case. Obviously, this construction will require a lot of statistics, which may be available only towards the end of the ILC run. A similar possibility is to produce a pair of $Z^{\prime}$ bosons in association with a $\ell^{+} \ell^{-}$pair, i.e. $e^{+} e^{-} \rightarrow Z^{\prime} Z^{\prime} \ell^{+} \ell^{-}$, which can arise from several diagram topologies, such as, e.g., $e^{+} e^{-} \rightarrow Z^{\prime} Z^{\prime} \gamma^{*}$ or $e^{+} e^{-} \rightarrow \ell^{+} \ell^{-}$with two radiated $Z^{\prime}$-s. One of the $Z^{\prime}$ bosons would then have to decay to neutrinos, while the remaining one decays to another $\ell^{+} \ell^{-}$pair (which will exhibit the invariant mass peak). Yet another possibility is $e^{+} e^{-} \rightarrow Z Z^{\prime} \ell^{+} \ell^{-}$(for which again there are several diagram topologies) which can be treated similarly.

2. Another alternative is to produce a pair of heavy $W^{\prime \pm}$ bosons, with an ordinary $Z$ boson radiated from any of the fermion legs, i.e. $e^{+} e^{-} \rightarrow W^{\prime+} W^{\prime-} Z$. Each $W^{\prime}$ would decay to $\ell \nu_{\ell}$, while the $Z \rightarrow \ell^{+} \ell^{-}$(all daughter pairs having, in principle, different lepton flavours). Such heavy $W^{\prime}$ s are predicted in many theories, such as left-right symmetric models, 3-3-1 gauge models, and so on. As before, the leptons will tend to be hard and lead to elimination of these events by the $p_{T}^{\ell}$ cut, and it may again be possible to distinguish this case from the UED one by vetoing the events when an $\ell^{+} \ell^{-}$invariant mass peaks around $M_{Z}$ when enough statistics has been accumulated. An alternative process with $e^{+} e^{-} \rightarrow W^{\prime+} W^{\prime-} \ell^{+} \ell^{-}$can also be envisaged, where, for example, the $\ell^{+} \ell^{-}$ pair arises from an off-shell photon radiation. Obviously, in this case, there may be no identifiable peak in the $\ell^{+} \ell^{-}$invariant mass, as a result of which the signal would closely resemble the $4 \ell+\mathbb{E}_{T}$ signal from UED in all kinematic characteristics. A large part of this will be removed by the $p_{T}^{\ell}$ cut, but there may still be some irreducible part which cannot be distinguished from the UED signal.

3. A pair of heavy neutralinos $\widetilde{\chi}_{i}^{0} \widetilde{\chi}_{j}^{0}(i, j>1)$, each of which decays as $\widetilde{\chi}_{i}^{0} \rightarrow \ell \widetilde{\ell} \rightarrow \ell\left(\ell \widetilde{\chi}_{1}^{0}\right)$, where $\tilde{\ell}$ denotes a slepton. In a SUSY model where $R$-parity is conserved, the LSP $\widetilde{\chi}_{1}^{0}$ is invisible, so that we would eventually observe four leptons together with missing energy and momentum. If the mass gap between the $\widetilde{\chi}_{i}^{0}(i>1)$ and the $\widetilde{\chi}_{1}^{0}$ is not very large, the final state leptons can be expected to be reasonably soft - mimicking the UED signal almost perfectly. This, in fact, is an example of the similarities because of which the UED model was originally nicknamed 'bosonic SUSY'.

4. A pair of neutralinos $\widetilde{\chi}_{i}^{0} \widetilde{\chi}_{j}^{0}(i, j=1,4)$, each of which decays as $\widetilde{\chi}_{i}^{0} \rightarrow \widetilde{\ell \ell^{*}} \rightarrow \ell(\ell \nu)$ or as $\widetilde{\chi}_{i}^{0} \rightarrow \nu \widetilde{\nu}^{*} \rightarrow \nu(\ell \ell)$ in a SUSY model where $R$-parity is violated through $L L \bar{E}$ couplings. A similar possibility would be pair-production of charginos $\widetilde{\chi}_{i}^{ \pm} \widetilde{\chi}_{j}^{\mp}(i, j=1,2)$, where one decays to $\widetilde{\chi}_{i}^{ \pm} \rightarrow \ell^{ \pm} \widetilde{\nu}^{*} \rightarrow \ell^{ \pm}\left(\ell^{+} \ell^{-}\right)$and the other as $\widetilde{\chi}_{i}^{ \pm} \rightarrow \nu \widetilde{\ell}^{ \pm *} \rightarrow \nu\left(\ell^{ \pm} \nu\right)$. Other processs, such as production of a pair of sleptons (each decaying to a lepton and missing energy) in association with an $\ell^{+} \ell^{-}$pair, are sub-dominant. In all these cases, at least 
one of the final state leptons would tend to be much harder than in the UED case, and the upper cut $p_{T}^{\ell} \leq 40 \mathrm{GeV}$ may be enough to virtually eliminate the $R$-parityviolating alternatives at the ILC. The $p_{T}^{\ell} \leq 100 \mathrm{GeV}$ cut at the CLIC would still be a useful one, but probably much less efficient in removing this alternative.

5. In a model with large extra dimensions, it is possible to have several underlying SM diagrams (e.g. $Z Z$ production) ending in a $4 \ell$ final state in association with a tower of invisible $\mathrm{KK}$ gravitons radiated from any leg or vertex, e.g. $e^{+} e^{-} \rightarrow Z Z G_{n} \rightarrow$ $\left(\ell^{+} \ell^{-}\right)\left(\ell^{+} \ell^{-}\right) \mathbb{E}_{T}$. Some of these events will be removed by the $p_{T}^{\ell}$ cut, and some can be eliminated by vetoing events where the $\ell^{+} \ell^{-}$invariant mass peaks at $M_{Z}$, but there would remain a substantial irreducible background which may prove difficult to isolate from the UED signal.

The above list is illustrative, but not exhaustive. There exist possibilities galore, such as production of chargino pairs in association with $\ell^{+} \ell^{-}$, or processes involving graviton resonances, unparticles, and other exotica. As before, the best scenario would arise when we have accumulated a large number of events and more-or-less eliminated the extra $W^{\prime}, Z^{\prime}$ and graviton alternatives, while the $p_{T}^{\ell}$ cut has similarly eliminated the $R$-parity-violating version of SUSY. However, the third of the above alternatives, viz. heavy neutralino production and decay, is irreducible by kinematic means, and must still remain an alternative explanation of an excess in $4 \ell+\not E_{T}$ events.

Signals for UED at the ILC (or CLIC) represent, therefore, a typical case of the inverse problem, since there are several rival models contending for an explanation of each signature. The key to solving this must lie in considering more than one signal cross-section (or distribution) simultaneously [26]. Recent studies [27] of the inverse problem at the LHC suggest that it is convenient to define a 'signature space' of different signals, into which every point in the parameter space of new physics models will map uniquely. The map may not, however, be invertible, since the same signature may arise from different kinds of new physics, or even from different choices of parameters in the same model. Nevertheless, if different models map into different parts of the 'signature space', then the experimental data will pick up the correct model (or class of models) effortlessly. We now demonstrate that such could indeed be the case for the UED - and rival models - in the light of the signals discussed above.

In Figure 6 we have shown three such plots. The plot on the extreme left shows the UED parameter space discussed in the text. This may be thought of as a two-dimensional section of the multi-dimensional 'theory space' at ILC, which encompasses all new physics models and all their parameters. In the centre and on the right we show the 'signature space' at the ILC-2 and CLIC respectively, i.e. once again a two-dimensional section in the space of all 
possible signals, by plotting our predictions for the excess contributions (above SM) in the dijet and $4 \ell+\mathbb{E}_{T}$ cross-sections along the two axes in a plane. In order to generate these numbers the following kinematic cuts were used:

\section{- Dijets:}

(i) Each 'jet' must have transverse momentum $p_{T}^{\text {jet }} \geq 100 \mathrm{GeV}$.

(ii) Each 'jet' must have pseudorapidity $\left|\eta_{\text {jet }}\right| \leq 2.5$.

(iii) The dijet invariant mass must satisfy $M_{\mathrm{JJ}} \geq 600 \mathrm{GeV}$.

(iv) The angular separation between the jets must satisfy $\Delta R_{\mathrm{JJ}} \geq 0.7$.

- $4 \ell+E_{T}$ :

(i) Each lepton must have transverse momentum $5 \mathrm{GeV} \leq p_{T}^{\ell} \leq 40 \mathrm{GeV}$ at the ILC-2. At the CLIC, the corresponding cuts will be $10 \mathrm{GeV} \leq p_{T}^{\ell} \leq 100 \mathrm{GeV}$.

(ii) Each lepton must have pseudorapidity $\left|\eta_{\ell}\right| \leq 2.5$.

(iii) The angular separation between the leptons must satisfy $\Delta R_{\ell \ell} \geq 0.2$.

(iv) The missing transverse energy in the system must satisfy $E_{T} \geq 100 \mathrm{GeV}$.

$(v)$ Dileptons of the same flavour in the final state must not have invariant mass $M_{\ell \ell}$ in the range $80-100 \mathrm{GeV}$. (This is essentially a veto on leptons coming from the decay of a $Z$-boson.)

In all three plots in Figure 6, the following conventions are used:

- The solid (black) lines represent constant values of $\Lambda R$, with $R^{-1}$ increasing along the direction of the arrows. It may be noted that the arrow direction is reversed when we go from the central box (ILC-2) to the right box (CLIC). The reasons are explained below.

- The dashed (blue) lines represent constant values of $R^{-1}$, with $\Lambda R$ increasing along the direction of the arrows.

- The thick solid (blue) line represents the experimental constraint $R^{-1} \gtrsim 300 \mathrm{GeV}$.

- The vertical (black) line in the box on the extreme left around $R^{-1}=500 \mathrm{GeV}$ (which ends in dashes) represents the kinematic reach of the ILC-2. In the plot in centre, this lies right on the horizontal axis. It is not shown on the CLIC plot, on the right.

- The thick vertical (blue) hatched line in the left box represents the dark matter constraint $R^{-1} \lesssim 900 \mathrm{GeV}$. This is rendered hatched rather than solid to indicate that there is a theoretical bias in this constraint. There is no such line in the central box because the ILC-2 cannot access this part of the parameter space. On the extreme right this bound appears very close to the axis. 
- The other (red) hatched lines represent the limits on $\Lambda R$, viz. $\Lambda R \gtrsim 5$ and $\Lambda R \lesssim 50$. Once again, the hatching represents the fact that there is a theoretical bias in these constraints - we may recall that these are essentially ballpark values.

- In the (signature space) plots in the centre and on the right, the solid (red) blob at the origin represents the SM prediction, as marked. The size of the blob is a very rough indicator of the error level (at $1 \sigma$ ) if the integrated luminosity reaches around $100 \mathrm{fb}^{-1}$. We assume this ballpark figure both for the ILC-2 and for the CLIC. This tiny blob indicates that the UED model will predict a sizable excess in both crosssections, irrespective of the parameters chosen.
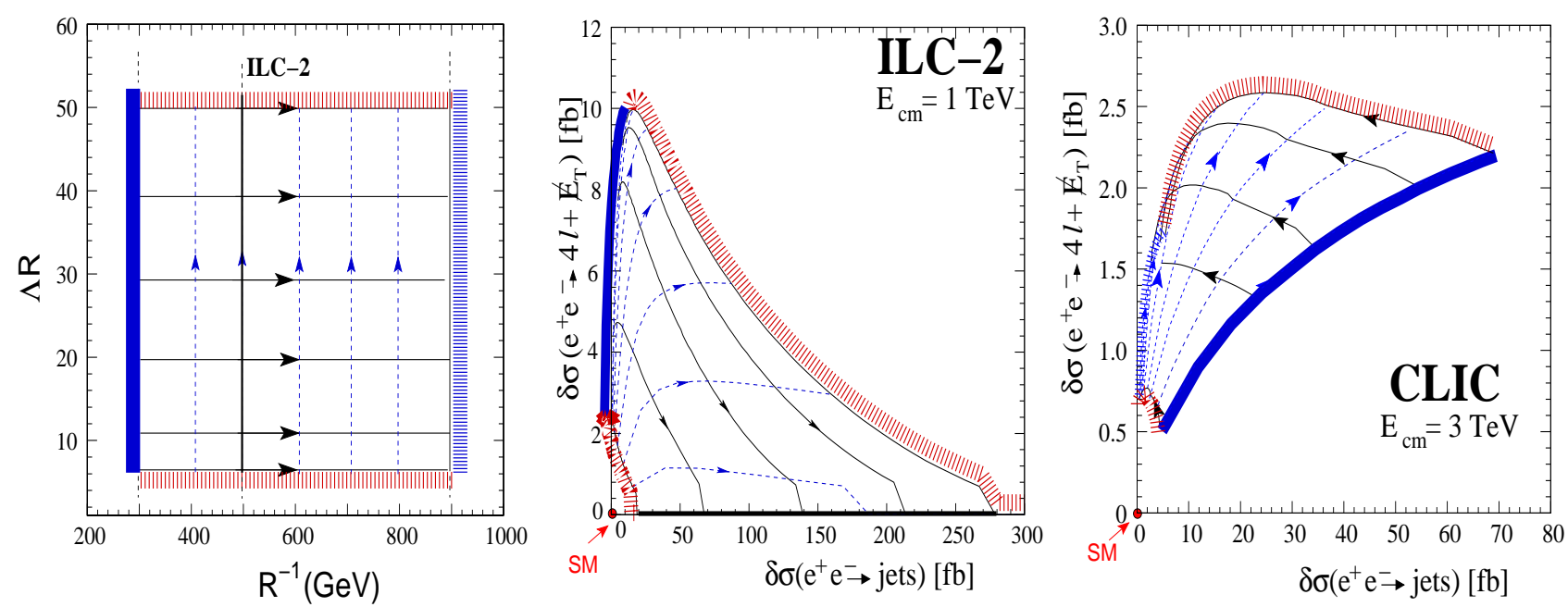

Figure 6: Illustrating the correlation of two different cross-sections to obtain a distinctive signature space for UED at the ILC-2 and the CLIC. The parameter space is shown in the box on the extreme left, while the excess (over SM) cross-sections are shown in the central box for the ILC-2 and on the extreme right for the CLIC. Other conventions are explained in the text.

Figure 6 has several interesting features. Let us first consider the central box, i.e. the ILC-2 plot. We note that this plot is relevant for only the part of the parameter space where $300 \mathrm{GeV}<R^{-1}<500 \mathrm{GeV}$, i.e., which is kinematically accessible at the ILC-2. As $R^{-1}$ increases towards the kinematic limit of $500 \mathrm{GeV}$, the excess cross-section for $4 \ell+\mathbb{E}_{T}$ falls quite sharply to the SM value (the origin on the plot) while the dijet cross-section keeps growing because the increase in electron flux as $x \rightarrow 1$ is strong enough to mask phase space effects. Of course, once the resonant value is crossed, the dijet cross-section immediately shrinks back almost to the origin, i.e. the SM value. This is not visually apparent in Figure 6 because that would require a very fine scale on the vertical axis, but we have checked numerically that this is indeed what happens. An indicator of this phenomenon is provided by the 'knee' noticeable in each curve close to the horizontal axis, which represents 
a point where the heavier $Z_{2}$ resonance is pushed out of the kinematic range of the ILC-2, while the lighter $\gamma_{2}$ resonance is still accessible through radiative effects.

The box on the extreme right shows a similar plot at the CLIC, where the most interesting feature is that some of the arrows have reversed sign, indicating a fall in the cross-section as $R^{-1}$ increases. This is not really a surprise, however. At the CLIC, running at $\sqrt{s}=3 \mathrm{TeV}$, the allowed range $R^{-1}=0.3-0.9 \mathrm{TeV}$, indicates, that the electrons and positrons producing a resonance will typically carry momentum fractions in the ballpark of 0.1 to 0.3 , for which Figure 3 tells us that the luminosity function is essentially flat. Since the maximum mass of resonances in this range of $R^{-1}$ will be less than $2 \mathrm{TeV}$, and the machine energy is $3 \mathrm{TeV}$, there will be no dramatic effects due to phase space either. Thus, the resonant cross-section will essentially echo the behaviour of the propagator, which, in the narrow-width approximation can be written as

$$
\frac{1}{\left(\hat{s}-M_{2}^{2}\right)^{2}+M_{2}^{2} \Gamma_{2}^{2}} \approx \frac{1}{M_{2} \Gamma_{2}} \delta\left(\hat{s}-M_{2}^{2}\right),
$$

where $M_{2}$ stands for the mass of the $\gamma_{2}$ or the $Z_{2}$, as the case may be. The delta function is, of course responsible for setting the value of $x_{1} x_{2}$. Noting that $M_{2} \gtrsim 2 R^{-1}$ and that $\Gamma_{2}$ increases as $M_{2}$ increases, it becomes clear that the resonant cross-section will fall as $R^{-1}$ increases. It is this effect which is reflected in the direction of the arrows on the solid lines in the CLIC plot. We reiterate that at the ILC-2 this effect, though present, gets masked by the enormous increase in luminosity as $R^{-1}$ increases over the small range permissible at this machine.

A glance at the rough error estimate on the SM points indicated in Figure 6 shows that if the value of $R^{-1}$ is kinematically accessible, a clear signal for new physics may be obtained at the ILC-2 and CLIC, for a wide range of parameters of the UED model. The question one may then ask is whether this signal can be uniquely identified as due to a UED. In the earlier part of this section we have seen a sample of new physics models which could yield similar signals. We now contend that these will have different characteristics when mapped on to the signature space illustrated (for ILC-2 and CLIC) in Figure 6. In order to establish this argument, we take up these alternative models one at a time.

- Extra neutral gauge bosons: As we have argued above, at the ILC-2 we can easily have a $4 \ell+E_{T}$ signal from the production of a pair of $Z^{\prime}$ bosons in association with a radiated photon $\gamma^{*}$ or a $Z$ boson. This would, obviously, happen at the ILC-2 only if $M_{Z^{\prime}}<\sqrt{s} / 2 \simeq 500 \mathrm{GeV}$. The $Z^{\prime}$ could also form a resonance9 in the $e^{+} e^{-} \rightarrow J J$ cross-section, and hence appear as a point in the signature space overlapping with the portion occupied by the UED signal. However, at the ILC-2, the position of the

\footnotetext{
${ }^{9}$ Or even a pair of resonances, if there is a nearly-degenerate $Z^{\prime \prime}$.
} 
resonance in the invariant mass plot shown in Figure 4 would be a dead giveaway, since any $n=2$ resonance in UED will lie approximately at $\sqrt{s} \approx 2 R^{-1} \gtrsim 600 \mathrm{GeV}$, whereas the $Z^{\prime}$ resonance must perforce lie at $\sqrt{s}=M_{Z^{\prime}}<500 \mathrm{GeV}$.

A more interesting possibility at the ILC-2 is a single heavy $Z^{\prime}$ boson, with $M_{Z^{\prime}}>$ $500 \mathrm{GeV}$, which not only creates a resonance in the dijet cross-section, but is also produced in association with a lepton pair and an ordinary $Z$ boson. For example, we can have a standard $e^{+} e^{-} \rightarrow \ell^{+} \ell^{-}$process, with both the $Z$ and the $Z^{\prime}$ radiated off the leptonic legs. Now, if the $Z^{\prime}$ boson decays invisibly $\left(Z^{\prime} \rightarrow \nu \bar{\nu}\right)$, and the $Z$ decays to a $\ell^{+} \ell^{-}$pair, there may be a significant number of events with four soft $\left(p_{T}^{\ell}<40 \mathrm{GeV}\right)$ leptons. In this case, however, at least two of the leptons will have an invariant mass peaking at $M_{Z}$, and this configuration would be eliminated by the cuts used to generate Figure 6. If, on the other hand, it is the $Z$ boson which decays invisibly, and the $Z^{\prime}$ which gives a dilepton, then there will be a peak in the invariant mass of at least one pair of leptons. This peak should match the resonant peak in the dijet cross-section, thus giving away their common $Z^{\prime}$ origin. This can be used to discriminate from the UED model, where there should be no peaking in $M_{\ell \ell}$, and where the dijet resonances are roughly twice as heavy as the $Z_{1}$. Pinning down a UED model at the ILC-2 may, therefore, have to wait until enough statistics is accumulated to make an unambiguous statement about the presence or absence of a peak in the invariant mass spectrum of all the possible lepton pairings.

At the CLIC, running at $\sqrt{s}=3 \mathrm{TeV}$, a $Z^{\prime}$ pair can be produced so long as $M_{Z^{\prime}}<$ $\sqrt{s} / 2 \simeq 1.5 \mathrm{TeV}$. This invalidates the argument used above for the ILC-2, since we could very well have a $Z^{\prime}$ in the mass range $600 \mathrm{GeV}$ to $1.5 \mathrm{TeV}$, which can be pairproduced as well as excite a resonance. Moreover, the larger bin-size at the CLIC would make it more likely for a single resonant peak to be observed. The signals from a model with a $Z^{\prime}$ boson could, therefore, mimic the UED signal in every particular, except for one, viz. the fact already pointed out above: that in the $4 \ell+\mathbb{E}_{T}$, at least one pair of leptons will show an invariant mass peak corresponding to the $Z^{\prime}$ boson mass - and this will match the resonant value of dijet invariant mass. Presumably the higher luminosity of the CLIC would make it easy to make an early identification of peaks in the dilepton invariant mass as envisaged here.

- Extra charged gauge bosons: We have argued earlier, that the production of a pair of heavy $W^{\prime \pm}$ gauge bosons in association with an $\ell^{+} \ell^{-}$pair can closely mimic the $4 \ell+E_{T}$ signal from UED. However, in this case, no resonance will be seen in the dijet invariant mass, simply because the initial and final states are charge-neutral. The observed point in the signature space shown in Figure [6 will lie somewhere on the vertical axis, and 
hence, be clearly distinct from a UED signal. This will be as true at the CLIC as at the ILC-2.

It is legitimate to ask the question: what will happen if there are both $Z^{\prime}$ resonances as well as $W^{\prime}$ states which can be pair-produced in $e^{+} e^{-}$collisions? Such combinations of states do exist in, for example, left-right symmetric models. At the ILC-2, existing constraints on such models preclude pair-producion of the extra charged gauge bosons, but at the CLIC, this may be a real possibility. It is true that in most of the existing models the $W^{\prime}$ would be more-or-less as heavy as the $Z^{\prime}$ (if not heavier) and not about half as massive as is envisaged in this work 10 . However, if we confine ourselves to a signature space of $4 \ell+E_{T}$ signals versus dijet signals, any such model will certainly yield nonzero results for both. Fortunately, the leptonic decays of a heavy $W^{\prime}$ will tend to produce hard leptons which would again fall foul of the $p_{T}^{\ell}<40$ (100) GeV cut imposed on the $4 \ell+E_{T}$ signal at the ILC-2 (CLIC). Ultimately, therefore, this veto on hard leptons will remove a large part of the cross-section and thus it may be expected these models will map to points very close to (but not exactly on) the abscissa in the signature space of Figure 6.

- Massive gravitons: Let us first consider the case of large extra dimensions [28]. We have seen that a tower of invisible but massive graviton states can closely mimic the UED model for the $4 \ell+E_{T}$ signal. However, when we consider the $e^{+} e^{-} \rightarrow J J$ process, there should be an excess cross-section, but no identifiable resonance(s) - the excess cross-section forming a continuum in invariant mass [29]. If the technique adopted to identify a resonance is not inspection, but, for example, a $3 \sigma$ deviation from the SM in one or more bins of $M_{J J}$, it should be designed with a veto on an excess cross-section which spreads across several bins. Since, the horizontal axis in Figure 6 is plotted assuming a resonance, we can assume the excess resonant $e^{+} e^{-} \rightarrow J J$ cross-section to vanish, so that the large extra dimensions model will eventually predict a point lying on the vertical axis.

If we consider a single heavy graviton $G_{1}$, as predicted in the Randall-Sundrum model [30], a resonance in the $e^{+} e^{-} \rightarrow J J$ cross-section is predicted, and this will resemble the UED case when there are overlapping $\gamma_{2}, Z_{2}$ peaks. Such a graviton state can also be produced in association with a $4 \ell$ state, and decay invisibly as $G_{1} \rightarrow \nu \bar{\nu}$. Equivalently, it can be produced in association with an $\ell^{+} \ell^{-} \mathbb{E}_{T}$ signal and itself decay to another $\ell^{+} \ell^{-}$pair. In either case we obtain a $4 \ell+E_{T}$ signal. Thus, the presence of a massive graviton $G_{1}$ will produce an overlap with the UED model in the signature

\footnotetext{
${ }^{10}$ This is not to say that one cannot devise a model in which this feature appears - even if it is done only to play the Devil's advocate.
} 
space of Figure 6. There are now two ways to distinguish it from the UED signal, both requiring a fairly large number of events. A purely kinematic method would be to study the invariant mass distribution of every lepton pair in the $4 \ell+\mathbb{E}_{T}$ final state. Obviously, for all the events involving a graviton decay $G_{1} \rightarrow \ell^{+} \ell^{-}$, the invariant mass will cluster around $M_{G_{1}}$, which should match with the resonance in $e^{+} e^{-} \rightarrow J J$. This matching cannot happen in the UED model, where the $Z_{1}$ bosons leading to missing energy and momentum are about half as massive as the $\gamma_{2}, Z_{2}$ resonances. Another method would be to reconstruct the angular distribution $d \sigma / d\left(\cos \theta^{*}\right)$ of the dijet states in the centre-of-mass frame and see if they exhibit the characteristics of spin-1 particles, i.e. $\propto P_{1}\left(\cos \theta^{*}\right)$, or spin-2 particles, i.e. $\propto P_{2}\left(\cos \theta^{*}\right)$, where $P_{1,2}$ denote the Legendre polynomials of order 1 and 2 respectively. This second method will have errors arising from imperfections in jet reconstruction, but will gain vastly in the number of events when compared with the first, purely kinematic, method. Hence it would probably be the method of choice should a signal be seen in the early days of ILC-2, with the other method chipping in as a confirmatory test when enough statistics have accumulated.

- Supersymmetry with and without R-parity conservation: We have argued above that if $R$-parity is conserved, a $4 \ell+E_{T}$ final state can easily arise in SUSY from the production of a pair of heavy neutralinos decaying to the invisible LSP. However, the same conservation principle which renders the LSP invisible also precludes any of the heavy supersymmetric particles from appearing as resonances in $e^{+} e^{-} \rightarrow J J$. Hence, the predicted point will lie along the vertical axis in the signature space of Figure 6 .

The distinction becomes somewhat more difficult if $R$-parity is violated, for then we can have sneutrino resonances in the $e^{+} e^{-} \rightarrow J J$ cross-section as well as an excess in the $4 \ell+E_{T}$ signal due to a pair of decaying neutralino LSPs. Here, as in the case of a massive graviton resonance, we can use a purely kinematic method and/or a dynamical method relying on the fact that the sneutrino is a scalar. The dynamical method is quite simple, since it involves the same construction of $d \sigma / d\left(\cos \theta^{*}\right)$ for the dijet final state as described above. If the exchanged particle is a scalar, this should be $\propto P_{0}\left(\cos \theta^{*}\right)$, i.e. constant (apart from kinematic effects near $\cos \theta= \pm 1$ which are due to the acceptance cuts). Any substantial variation in $d \sigma / d\left(\cos \theta^{*}\right)$ should immediately rule out the sneutrino hypothesis. At the same time, we may note that the kinematic cut $p_{T}^{\ell} \leq 40 \mathrm{GeV}$ can also remove most of the $4 \ell+E_{T}$ signal at the ILC for an LSP mass of around $100 \mathrm{GeV}$ or more. The corresponding cut, $p_{T}^{\ell} \leq 100 \mathrm{GeV}$ at the CLIC will be less efficient, but would still reduce the LSP decay signal considerably. Assuming this to be the case, the predicted point in the signature space will lie practically on the horizontal axis. At the ILC-2, this will be similar to the UED prediction with $R^{-1} \lesssim 500 \mathrm{GeV}$, but, of course, when we go to the CLIC, the UED prediction will no 
longer be on the horizontal axis (as shown in the right-most box in Figure 6), whereas that from $R$-parity-violating SUSY will continue to be so. One or other of the two methods will suffice to pick out UED from $R$-parity-violating SUSY.

The above list of models is illustrative, but by no means exhaustive11. It may happen that the reality at the $\mathrm{TeV}$ scale comprises not just one of the above models, but a combination of two or more of these. For example, if we have a supersymmetric model with an extended gauge sector, i.e. an extra $Z^{\prime}$ boson, we may expect an excess in the $4 \ell+\mathbb{E}_{T}$ signal due to the production of a slepton pair in association with a $Z$ or a $Z^{\prime}$ boson which then decays to leptons. At the same time, the $Z^{\prime}$ could give rise to resonances in the dijet cross-section. However, some of the tests suggested above could still be used to distinguish this scenario from the UED case. For example, the leptons coming from the $Z^{\prime}$ decay would not only tend to be hard, unlike those coming from a $Z_{1}$ to $\ell_{1}$ to $\gamma_{1}$ cascade decay, but would also exhibit an invariant mass peak at the $Z^{\prime}$ mass which would match with the resonant mass of the dijets. Thus, the arguments presented above serve to illustrate the fact that it is indeed very difficult to create a model which would not have some kinematic or dynamical difference from the UED model so far as its signals at the ILC-2 go, especially when we combine two or more signals, and add on a spin measurement. It follows that if UEDs are indeed the new physics to be discovered at the next generation of accelerators, then it should be possible to make a unique statement to that effect, using some very simple kinematic constructions and physical arguments.

\section{Summary and Conclusions}

We have already stressed in the Introduction that the LHC does not lend itself readily to UED searches. It is likely that UED searches at the LHC would require considerable ingenuity and the collection of a large amount of data before any definitive conclusion can be reached. The strategies discussed in this paper have, therefore, deliberately been kept independent of the possible discovery of UED-s or some other form of new physics at the LHC - partly because it would be mere speculation to talk of a post-LHC scenario at the present juncture, and partly because we feel that the ILC (or CLIC) should be considered as an independent experiment in its own right.

In this work, we have focussed on the efficacy of radiation from the colliding beams in an $e^{+} e^{-}$

\footnotetext{
${ }^{11}$ There are already many other suggested models in the literature, such as little Higgs models with $T$ parity, unparticles, etc., which could also, in principle, have been included in the argument. Indeed, it would be foolhardy to try and make an exhaustive list, for that would not only be underestimating human ingenuity, but also be making the hubristic assumption that Nature cannot have any more surprises for us.
} 
collider in producing an energy spread and shown that this leads to enhanced cross-sections for resonant processes involving $n=2 \mathrm{KK}$ excitations of the photon and the $Z$-boson in a model with universal extra dimensions. For a few benchmark points, we have shown that one can get sharply-defined resonances in the invariant mass of final-state dijets. Focussing on these, and combining with the pair production of $Z_{1}$ resonances (followed by leptonic decays of the $Z_{1}$-s), we have shown how to create a two-dimensional 'signature space' and use it to distinguish UED signals from that of other, competing models for new physics. As we have seen, there is no 'smoking gun' signal which can be uniquely identified as due to UEDs, but the simple two-dimensional signature space of Figure 6, with at most, a spin measurement added, would be more-or-less sufficient to identify an underlying UED model uniquely.

A clear UED signal, corresponding to a point in the signature space well away from any of the axes would immediately tell us the values of the parameters $R^{-1}$ and $\Lambda R$, as corresponding to a unique point in the grid of Figure 6. It is, indeed, quite a remarkable feature of the UED model that almost complete disambiguation can be achieved with so simple an analysis, especially when we compare it with the intricate and computation-intensive methods which have been employed to study SUSY and string-inspired models [27]. While our analysis admittedly lacks sophistication in the reconstruction of jets, we contend that for a fairly high number of events, the predictions of a more elaborate simulation at an $e^{+} e^{-}$collider would closely resemble the ones presented in this paper. Moreover, we have chosen two final states, viz., dijets and $4 \ell$ plus missing $p_{T}$, for which data will certainly be collected and stored at any high energy $e^{+} e^{-}$machine. The search for and identification of UED-s can, therefore, be carried out without any extra cost, as it were, in data storage or even manpower, using the techniques described in this paper. Our analysis thus has the twin virtues of economy and simplicity. On that positive note, we end the discussion.

Acknowledgments: The authors would like to thank the organisers of LCWS-06 (Bangalore, 2006) and WHEPP-X (Chennai, 2008) where some of this work was done. We acknowledge partial financial support from the University Grants Commission, India (BB), the Department of Science and Technology, Government of India (AK), the Board of Research in Nuclear Science, Government of India (AK) and, finally, the Academy of Finland (SKR).

\section{References}

[1] For a fascinating account of the early history of extra dimensions, see, for example, L. O'Raifeartaigh and N. Straumann, arXiv:hep-ph/9810524.

[2] T. Appelquist, H. C. Cheng and B. A. Dobrescu, Phys. Rev. D 64 (2001) 035002. 
[3] See, for example, T. Appelquist, A. Chodos and P.G.O. Freund, Modern Kaluza-Klein Theories (Addison-Wesley, 1987).

[4] H. C. Cheng, K. T. Matchev and M. Schmaltz, Phys. Rev. D 66 (2002) 036005.

[5] H. C. Cheng, K. T. Matchev and M. Schmaltz, Phys. Rev. D 66 (2002) 056006.

[6] A. J. Barr, Phys. Lett. B 596 (2004) 205;

J. M. Smillie and B. R. Webber, JHEP 0510 (2005) 069.

[7] A. Datta, K. Kong and K. T. Matchev, Phys. Rev. D 72 (2005) 096006 [Erratum-ibid. D 72 (2005) 119901].

[8] B. Bhattacherjee et al., in preparation.

[9] B. Bhattacherjee and A. Kundu, Phys. Lett. B 627 (2005) 137.

[10] R. M. Godbole, S. K. Rai and S. Raychaudhuri, Eur. Phys. J. C 50 (2007) 979.

[11] G. Servant and T. M. P. Tait, Nucl. Phys. B 650 (2003) 391;

M. Kakizaki, S. Matsumoto, Y. Sato and M. Senami, Nucl. Phys. B 735 (2006) 84;

M. Kakizaki, S. Matsumoto and M. Senami, Phys. Rev. D 74 (2006) 023504;

D. Hooper and S. Profumo, Phys. Rept. 453 (2007) 29, and references therein.

[12] K. Agashe, N. G. Deshpande and G. H. Wu, Phys. Lett. B 514 (2001) 309;

D. Chakraverty, K. Huitu and A. Kundu, Phys. Lett. B 558 (2003) 173;

J. F. Oliver, J. Papavassiliou and A. Santamaria, Phys. Rev. D 67 (2003) 056002;

A. J. Buras, M. Spranger and A. Weiler, Nucl. Phys. B 660 (2003) 225;

A. J. Buras, A. Poschenrieder, M. Spranger and A. Weiler, Nucl. Phys. B 678 (2004) 455 .

[13] U. Haisch and A. Weiler, Phys. Rev. D 76 (2007) 034014.

[14] I. Gogoladze and C. Macesanu, Phys. Rev. D 74 (2006) 093012;

T. Flacke, D. Hooper and J. March-Russell, Phys. Rev. D 73 (2006) 095002 [Erratumibid. D 74 (2006) 019902].

[15] H. Georgi, A. K. Grant and G. Hailu, Phys. Lett. B 506 (2001) 207.

[16] M. Puchwein and Z. Kunszt, Annals Phys. 311 (2004) 288.

[17] I. Antoniadis, Phys. Lett. B 246 (1990) 377;

C. Macesanu, C. D. McMullen and S. Nandi, Phys. Rev. D 66 (2002) 015009;

C. Macesanu, C. D. McMullen and S. Nandi, Phys. Lett. B 546 (2002) 253; 
H. C. Cheng, Int. J. Mod. Phys. A 18 (2003) 2779;

A. Muck, A. Pilaftsis and R. Ruckl, Nucl. Phys. B 687 (2004) 55.

[18] G. Bhattacharyya, P. Dey, A. Kundu and A. Raychaudhuri, Phys. Lett. B 628 (2005) 141 ;

M. Battaglia, A. Datta, A. De Roeck, K. Kong and K. T. Matchev, JHEP 0507 (2005) 033.

[19] B. Bhattacherjee and A. Kundu, J. Phys. G 32 (2006) 2123;

B. Bhattacherjee and A. Kundu, Phys. Lett. B 653 (2007) 300;

S. K. Rai, Pramana 69 (2007) 815;

B. Bhattacherjee, Pramana 69 (2007) 855;

A. Datta and S. K. Rai, Int. J. Mod. Phys. A 23 (2008) 519;

S. K. Rai, Int. J. Mod. Phys. A 23 (2008) 823.

[20] See, for example, E. Accomando et al. [CLIC Physics Working Group], arXiv:hep-ph/0412251.

[21] M. Drees and R. M. Godbole, Z. Phys. C 59 (1993) 591.

[22] K. Yokoya and P. Chen, Lect. Notes Phys. 400 (1992) 415.

[23] A. Pukhov, arXiv:hep-ph/0412191.

[24] J. Brau et al., ILC Reference Design Report, Vol. 1, SLAC Report SLAC-R-857 (2007); A. Djouadi, J. Lykken, K. Monig, Y. Okada, M. J. Oreglia and S. Yamashita, arXiv:0709.1893 [hep-ph].

[25] T. G. Rizzo, Phys. Rev. D 64 (2001) 095010.

[26] S. Kumar Rai and S. Raychaudhuri, JHEP 0310 (2003) 020;

S. Dutta, P. Konar, B. Mukhopadhyaya and S. Raychaudhuri, Phys. Rev. D 68 (2003) 095005 .

[27] N. Arkani-Hamed, G. L. Kane, J. Thaler and L. T. Wang, JHEP 0608 (2006) 070;

G. L. Kane, P. Kumar and J. Shao, J. Phys. G 34 (2007) 1993;

C. F. Berger, J. S. Gainer, J. L. Hewett, T. G. Rizzo and B. Lillie, arXiv:0711.1374 [hep-ph].

[28] N. Arkani-Hamed, S. Dimopoulos and G. R. Dvali, Phys. Lett. B 429 (1998) 263;

I. Antoniadis, N. Arkani-Hamed, S. Dimopoulos and G. R. Dvali, Phys. Lett. B 436 (1998) 257;

N. Arkani-Hamed, S. Dimopoulos and G. R. Dvali, Phys. Rev. D 59 (1999) 086004. 
[29] T. Han, J. D. Lykken and R. J. Zhang, Phys. Rev. D 59 (1999) 105006;

G. F. Giudice, R. Rattazzi and J. D. Wells, Nucl. Phys. B 544 (1999) 3.

[30] L. Randall and R. Sundrum, Phys. Rev. Lett. 83 (1999) 3370. 